\title{
LARGE DEVIATIONS OF THE ENTROPY PRODUCTION RATE FOR A CLASS OF GAUSSIAN PROCESSES
}

\author{
AMARJIT BUDHIRAJA, YONG CHEN, AND LIHU XU
}

ABSTRACT. We prove a large deviation principle (LDP) and a fluctuation theorem (FT) for the entropy production rate (EPR) of the following $d$ dimensional stochastic differential equation

$$
\mathrm{d} X_{t}=A X_{t} \mathrm{~d} t+\sqrt{Q} \mathrm{~d} B_{t}
$$

where $A$ is a real normal stable matrix, $Q$ is positive definite, and the matrices $A$ and $Q$ commute. The rate function for the EPR takes the following explicit form:

$$
I(x)= \begin{cases}x \frac{\sqrt{1+\ell_{0}(x)}-1}{2}+\frac{1}{2} \sum_{k=1}^{d}\left(\sqrt{\alpha_{k}^{2}-\beta_{k}^{2} \ell_{0}(x)}+\alpha_{k}\right), & x \geq 0, \\ -x \frac{\sqrt{1+\ell_{0}(x)}+1}{2}+\frac{1}{2} \sum_{k=1}^{d}\left(\sqrt{\alpha_{k}^{2}-\beta_{k}^{2} \ell_{0}(x)}+\alpha_{k}\right), & x<0,\end{cases}
$$

where $\alpha_{k} \pm \mathrm{i} \beta_{k}$ are the eigenvalues of $A$, and $\ell_{0}(x)$ is the unique solution of the equation:

$$
|x|=\sqrt{1+\ell} \times \sum_{k=1}^{d} \frac{\beta_{k}^{2}}{\sqrt{\alpha_{k}^{2}-\ell \beta_{k}^{2}}}, \quad-1 \leq \ell<\min _{k=1, \ldots, d}\left\{\frac{\alpha_{k}^{2}}{\beta_{k}^{2}}\right\} .
$$

Simple closed form formulas for rate functions are rare and our work identifies an important class of large deviation problems where such formulas are available. The logarithmic moment generating function (the fluctuation function) $\Lambda$ associated with the LDP is given as

$$
\Lambda(\lambda)= \begin{cases}-\frac{1}{2} \sum_{k=1}^{d}\left(\sqrt{\alpha_{k}^{2}-4 \lambda(1+\lambda) \beta_{k}^{2}}+\alpha_{k}\right) & \lambda \in \mathcal{D}, \\ \infty, & \lambda \notin \mathcal{D},\end{cases}
$$

where $\mathcal{D}$ is the domain of $\Lambda$. The functions $\Lambda(\lambda)$ and $I(x)$ satisfy the Cohen-Gallavotti symmetry properties:

$$
\Lambda(x)=\Lambda(-(1+x)), \quad I(x)=I(-x)-x, \text { for all } x \in \mathbb{R} .
$$

In particular, the functions $I$ and $\Lambda$ do not depend on the diffusion matrix $Q$, and are determined completely by the real and imaginary parts of the eigenvalues of $A$. Formally, the deterministic system with $Q=0$ has zero EPR and thus the model exhibits a phase transition in that the EPR changes discontinuously at $Q=0$.

Keywords: Entropy production rate, Large deviation principle, Gallavotti-Cohen functional, Itô-Wiener chaos, Sturm-Liouville problems, Nonequilibrium statistical mechanics.

$\operatorname{MSC}(2010)$ : 60F10, 60H10, 82C05.

\section{INTRODUCTION}

Let $\left\{X_{t}\right\}$ be a Markov process with sample paths in $C([0, \infty) ; \mathcal{E})$ (space of continuous functions from $[0, \infty)$ to $\mathcal{E}$ equipped with the local uniform topology), where $\mathcal{E}$ is some Polish space, with a stationary distribution $\mu$. For $t>0$, denote by $\mathbb{P}_{[0, t]}^{\mu}$ the probability law on $C([0, t] ; \mathcal{E})$ (space of continuous functions from $[0, t]$ to $\mathcal{E}$ equipped with the uniform topology) of $\left\{X_{s}\right\}_{0 \leq s \leq t}$, under the stationary measure (i.e. when $X_{0}$ has distribution $\mu$ ). Also, denote by $\mathbb{P}_{[0, t]}^{\mu,-}$ the probability law of the time reversed process, namely $\mathbb{P}_{[0, t]}^{\mu,-}=\mathbb{P}_{[0, t]}^{\mu} \circ \theta_{t}^{-1}$, where $\theta_{t}: C([0, t] ; \mathcal{E}) \rightarrow C([0, t] ; \mathcal{E})$ is defined as $\theta_{t}(x)(s) \doteq x(t-s), 0 \leq s \leq t$. Sample entropy production rate, also known as the Gallavotti-Cohen functional, of the Markov process $\left\{X_{t}\right\}$ 
under the stationary distribution $\mu$ is defined as

$$
e_{p}(t)= \begin{cases}\frac{1}{t} \log \frac{\mathrm{dP}_{[0, t]}^{\mu}}{\mathrm{dP}_{[0, t]}^{\mu,-}} & \text { if } \mathbb{P}_{[0, t]}^{\mu} \ll \mathbb{P}_{[0, t]}^{\mu,-}, t>0 . \\ \infty & \text { otherwise }\end{cases}
$$

Note that $e_{p}(t)=0$ for all $t>0$ if and only if the Markov process is reversible under the stationary measure $\mu$. Thus EPR can be viewed as a measure of irreversibility of the process $\left\{X_{t}\right\}$. Entropy production as a means to quantify irreversibility of a physical process has been considered in a broad range of model settings e.g. chemical networks [7] and biological populations [35]; and across a wide spectrum of temporal and spatial scales, from cells to planetary climates [38]. The mathematical formulation of EPR for stochastic processes in terms of time reversed processes originated in [27, 29, 31]; see also [23, 28, 36, 8, 11]. For more recent work on asymptotics of entropy production rate, we refer the reader to [2, 34, 33, 21, 22] and the references therein. In this work we consider a $\mathbb{R}^{d}$-valued Markov process given by the solution of the stochastic differential equation (SDE):

$$
\mathrm{d} X_{t}=A X_{t} \mathrm{~d} t+\sqrt{Q} \mathrm{~d} B_{t}
$$

where $A \in \mathbb{R}^{d \times d}, Q \in \mathbb{R}^{d \times d}$ satisfy the following stability and irreducibility assumption:

(A) All the eigenvalues of $A$ have negative real parts and $Q$ is positive definite.

Under Assumption (A), $\left\{X_{t}\right\}$ admits a unique invariant measure $\mu$ and the empirical EPR process $e_{p}(t)$ is well defined. The first goal of this work is to establish a large deviation principle (LDP) for the EPR process $e_{p}(t)$ as $t \rightarrow \infty$. From the classical work of Donsker and Varadhan[6] (see also [3, 6, 26, 39]), the large deviation behavior of the empirical measure process $\frac{1}{t} \int_{0}^{t} \delta_{X_{s}} d s$ is well understood. However, the large deviations of the process $e_{p}(t)$ cannot be deduced from these results in a simple manner. In particular, this process is given in terms of empirical average of a quadratic functional of the state process together with a time averaged stochastic integral (see (2.2)). The superlinearity of the functional of interest makes the analysis of large deviation properties of the EPR particularly challenging.

A second objective of this work is to establish the 'fluctuation theorem' (FT) for the diffusion given in (1.1). A fluctuation theorem in non-equilibrium statistical mechanics, as formulated in the mathematical theory by Cohen and Gallavotti[11], is a symmetry property of the rate function $I$ associated with the LDP for the EPR process $e_{p}(t)$ which states that $I(x)+\frac{x}{2}=$ $I(-x)-\frac{x}{2}$, for all $x \in \mathbb{R}^{d}$. Formally speaking, such a property gives a universality result which says that, for large $T$, the ratio between the probabilities of events $\left\{e_{T}=x\right\}$ and $\left\{e_{T}=-x\right\}$ is close to a model independent quantity given as

$$
\frac{P\left[e_{T}=x\right]}{P\left[e_{T}=-x\right]} \approx \exp \{-T(I(x)-I(-x))\}=\exp \{T x\}
$$

In this work, the fluctuation theorem that gives the Cohen-Gallavotti symmetry properties of the rate function, for the model in (1.1), will be established by identifying an explicit closed form expression for the rate function (see (2.6)). Explicit formulas for rate functions are rare and our work identifies an interesting and important class of large deviation problems for which such formulas are available.

A special case of (1.1) was studied in Chen et al. [4] where a three dimensional Langevin equation governing the motion of a charged test particle in a constant magnetic field was analyzed. In this model $X_{t}=\left[X_{t}^{1}, X_{t}^{2}, X_{t}^{3}\right]^{\prime}$, with ' being the transpose operation, is the particle's velocity at the time $t ; B_{t}=\left[B_{t}^{1}, B_{t}^{2}, B_{t}^{3}\right]^{\prime}$ is a three dimensional standard Brownian motion, 
and

$$
A=-\left[\begin{array}{ccc}
\cos \theta & -\sin \theta & 0 \\
\sin \theta & \cos \theta & 0 \\
0 & 0 & \cos \theta
\end{array}\right], Q=\left[\begin{array}{ccc}
\cos \theta & 0 & 0 \\
0 & \cos \theta & 0 \\
0 & 0 & \cos \theta
\end{array}\right], \quad \theta \in\left(-\frac{\pi}{2}, \frac{\pi}{2}\right) .
$$

The coordinates $X_{t}^{3}$ and $\left[X_{t}^{1}, X_{t}^{2}\right]^{\prime}$ are respectively parallel and perpendicular to the magnetic field, and so the Lorentz force only acts on $\left[X_{t}^{1}, X_{t}^{2}\right]^{\prime}$, driving the test particle to spiral around the magnetic field line. The Brownian motion $B_{t}$ models the collisions between the test particle and the ones in the medium. The dissipation in (1.1), with a strength $\cos \theta$, models the "wave propagation' produced by particles collisions, and the amplitude and sign of $\sin \theta$ describe the strength and direction of the magnetic field respectively. Finally, the diffusion coefficient $\cos \theta$ is derived from the classical mean square displacement assumption in statistical mechanics. For additional details on the background of (1.1), we refer the reader to [1, Chapter 11]. We note that this three dimensional system satisfies the following magnetic field property:

$$
A A^{\prime}=A^{\prime} A, \quad A Q=Q A .
$$

This property plays a key role in the analysis. Since in the above three dimensional model $X_{t}^{3}$ is a reversible Ornstein-Uhlenbeck process (independent of the first two coordinates), whose EPR is always zero, it suffices, for studying the large deviation and fluctuation theorem for EPR of (1.1), to consider the simplified two dimensional system given as:

$$
\left[\begin{array}{l}
\mathrm{d} X_{t}^{1} \\
\mathrm{~d} X_{t}^{2}
\end{array}\right]=-\left[\begin{array}{cc}
\cos \theta & -\sin \theta \\
\sin \theta & \cos \theta
\end{array}\right]\left[\begin{array}{l}
X_{t}^{1} \\
X_{t}^{2}
\end{array}\right] \mathrm{d} t+\left[\begin{array}{cc}
\sqrt{\cos \theta} & 0 \\
0 & \sqrt{\cos \theta}
\end{array}\right]\left[\begin{array}{l}
\mathrm{d} B_{t}^{1} \\
\mathrm{~d} B_{t}^{2}
\end{array}\right] .
$$

A natural approach for the study of a large deviation principle for $e_{p}(t)$ is by an application of the Gärtner-Ellis Theorem [5] (see e.g. [2, 11, 21, 22]). A key step in the implementation of this approach is to compute the associated Cramér function and study its regularity properties. The paper [4] computed the Cramér function for the EPR associated with the above reduced two-dimensional problem by studying the Karhunen-Loève (KL) expansion of the complex valued stochastic process $z(t)=X_{t}^{1}+\mathrm{i} X_{t}^{2}$ [19]. In the current work we are interested in the general $d$-dimensional diffusion governed by (1.1) where the coefficients satisfy the stability and irreducibility condition in (A) and the magnetic field property (1.2). Analogous to the above three dimensional SDE for a single test particle in a magnetic field, the general $d$-dimensional equation in (1.1) can be viewed as a model for the dynamics of a collection of charged test particles in a constant magnetic field (cf. [1]). In this general setting a central tool in the proofs of [4], namely a Karhunen-Loève expansion for $X_{t}$ with independent stochastic coefficients is not available. Such an expansion was used in [4] (see equation (14) therein) in order to represent the (pre-limit) Cramér function (see (2.16)) in terms of an infinite product of the form

$$
\prod_{k \geq 1} \mathbb{E} e^{\frac{\lambda(1+\lambda)}{2} w_{k}^{2}}
$$

where $\lambda \in \mathbb{R}$ and $\left\{w_{k}\right\}$ is a sequence of independent normal random variables. This representation played a key role in [4] in the computation of the asymptotics of the Cramér function (see eg. [4, Corollary 3.3]). For the general multidimensional setting considered here, such a simple form representation cannot be given and therefore, here we take a different approach than the one based on a Karhunen-Loève expansion. The starting point in this approach is to decompose the integral of the quadratic form that appears in the exponent for the (pre-limit) Cramér function (see (2.16)) into a Wiener-Itô chaos expansion up to the second order. The representation that we obtain for the Cramér function (see Theorem 3.3) is significantly more involved than the representation in terms of independent normal random variables in (1.4) used in [4] and the study of its asymptotics requires a careful analysis of the spectral properties of the integral 
operator in the second chaos of the Wiener-Itô expansion. Specifically, a key idea in the analysis is to decompose the symmetric compact operator, associated with the map $H_{\lambda, T}$ appearing in the second chaos (see Proposition 3.1), acting on the complex Hilbert space $L^{2}\left([0, T] ; \mathbb{C}^{d}\right.$ ), into $d$ operators on $L^{2}([0, T] ; \mathbb{C})$, and obtain estimates on its eigenvalues by solving a family of Sturm-Liouville problems. These estimates play a central role in characterizing the domain of finiteness of the Cramér functional. This characterization of the domain and an analysis of its boundary properties is the main ingredient in the application of the Gärtner-Ellis Theorem. We remark that Wiener-Itô chaos expansions to study properties of exponential functionals of Gaussian quadratic forms have been used in [16, 12] in the study of certain mathematical finance problems; however the analysis methods are quite different from those used in the current work. Also, analysis of Sturm-Liouville problems to study spectral properties of integral operators has along history, in particular, in a simpler context such an analysis was carried out in [4]. Specifically, [4] considers integral operators $K_{T}$ on $L^{2}([0, T] ; \mathbb{C}), T<\infty$, associated with a single kernel which is the covariance function of the complex Gaussian process $z(t)$, whereas here we need to analyze the spectral properties of integral operators on $L^{2}\left([0, T] ; \mathbb{C}^{d}\right)$ associated with an infinite collection of kernels $\left\{H_{\lambda, T}, \lambda \in \mathbb{R}, T<\infty\right\}$ (see Section 4).

A notable feature of our results is that, although the invariant measure of the Markov process in (1.1) obviously depends on $Q$, the Cramér function and the rate function for the LDP of $e_{p}(t)$ do not depend on this matrix (as long as Assumption (A) and (1.2) are satisfied). These quantities are completely determined from the real and imaginary parts of the eigenvalues of $A$, see Theorems 2.1 and 2.3. The noise term in (1.1) is usually thought of as external heat, with $Q$ describing its temperature, and the rate and Cramér functions $I$ and $\Lambda$ can be viewed as a type of entropy [6] and free energy [17], respectively. Our results say that as the temperature tends to zero, the entropy and the free energy quantities identified in Theorems 2.1 and 2.3 do not change. Formally, the deterministic system with $Q=0$, which is the zero trajectory, has zero EPR and thus the model exhibits a phase transition in that the EPR changes discontinuously at $Q=0$. It would be interesting to identify more general conditions under which a stochastic system exhibits such a phenomenon.

In a recent work Bertini et al. [2] study a $d$-dimensional diffusion process with diffusion coefficient $\sqrt{\epsilon}$ Id where $\epsilon$ is a small parameter. In view of the technical obstacles arising from the unboundedness (in fact quadratic growth when the drift is linear) of functionals describing the EPR process, they consider a setting where the drift $c$ admits a decomposition of the form $c=-\frac{1}{2} \nabla V+b$ where $b$ is a smooth and bounded vector field with bounded derivatives that is orthogonal to $\nabla V$ at every $x \in \mathbb{R}^{d}$. They propose a modified definition of the GallavottiCohen functional (i.e. the sample EPR) that is given in terms of the bounded vector field $b$ (see (1.7) therein). One of the main results in [2] establishes a large deviation principle for the modified Gallavotti-Cohen functional, as $\epsilon \rightarrow 0$ and $T \rightarrow \infty$ (in that order), which is described in terms of the Freidlin-Wentzell quasipotential associated with the small noise asymptotics of the diffusion [9]. Although, unlike the current work, [2] allows for nonlinear drifts, their analysis, due to the boundedness of $b$, becomes more tractable in some ways as they do not need to handle terms with a quadratic growth in the EPR process.

The recent works of Jakšić et al. [21, 22] prove an LDP for the entropy production for a family of Gaussian dynamical systems. Their proofs are analytic, relying on the properties of the maximal solution of a one-parameter family of algebraic matrix Riccati equations, rather than the tools from stochastic analysis such as chaos decomposition that are used in our work. In particular, their results do not provide explicit closed form formulas for the rate function of the type in (2.6).

The paper is organized as follows. Section 2 introduces some notation and definitions that are frequently used in this work and presents our main results. Sections 3 and 4 provide the 
analysis of the integral of the quadratic form that appears in the pre-limit Cramér function (see (2.16)) and that of the eigenvalues of the symmetric compact operator associated with the map $H_{\lambda, T}$ in the chaos expansion of this integral (see Proposition 3.1), respectively. Based on these preparatory results, we prove the LDP and the FT for the EPR in Section 5. The Appendix contains the elementary proof of the fact that as a consequence of (1.2)

(1.5)

the collection of matrices $\mathbb{M} \doteq\left\{A, A^{\prime}, Q, Q^{1 / 2}, Q^{-1}, Q^{-1 / 2}, M, N\right\}$ is a commuting family,

namely, for any $F, G \in \mathbb{M}, F G=G F$. Here

$$
M=A+A^{\prime}, \quad N=A-A^{\prime} .
$$

\section{ENTROPY PRODUCTION RATE AND MAIN RESULT}

2.1. Definition of EPR and the main results. Throughout this work we assume that Assumption (A) and the magnetic field property (1.2) are satisfied. Recall from Section 1 that under Assumption $\mathbf{A}, X_{t}$ admits a unique invariant measure $\mu$. In fact this measure is absolutely continuous with respect to Lebesgue measure and has the following density function:

$$
\rho(x)=(2 \pi)^{-\frac{d}{2}}(\operatorname{det} \Gamma)^{-\frac{1}{2}} \exp \left(-\frac{x^{\prime} \Gamma^{-1} x}{2}\right),
$$

with

$$
\Gamma=\int_{0}^{\infty} e^{A s} Q e^{A^{\prime} s} \mathrm{~d} s=-Q M^{-1}
$$

where the last equality is by (1.5) from which it follows that $A Q=Q A$ and $A^{\prime} Q=Q A^{\prime}$, and by using the fact that $M$ is negative definite (cf. [37]).

Denote by $\left(X_{t}^{\mu}\right)_{t \geq 0}$ the stationary process obtained by solving the SDE (1.1) with the random variable $X_{0}$ distributed according to $\mu$. For $0 \leq s<t<\infty$, consider the process $\left(X_{u}^{\mu}\right)_{s \leq u \leq t}$ and its time reversal $\left(X_{u}^{\mu,-}\right)_{s \leq u \leq t}:=\left(X_{t+s-u}^{\mu}\right)_{s \leq u \leq t}$. Note that both are stationary Markov processes. Let $\mathbb{P}_{[s, t]}^{\mu}$ and $\mathbb{P}_{[s, t]}^{\mu,-}$ be the distributions of $\left(X_{u}^{\mu}\right)_{s \leq u \leq t}$ and $\left(X_{u}^{\mu,-}\right)_{s \leq u \leq t}$.

Occasionally, for notational simplicity, we will write

$$
X_{t}=X_{t}^{\mu}, \quad X_{t}^{-}=X_{t}^{\mu,-}, \quad t>0 .
$$

From [23], the sample EPR of Eq. (1.1) is

$$
\begin{aligned}
e_{p}(t): & =\frac{1}{t} \log \frac{\mathrm{d} \mathbb{P}_{[0, t]}^{\mu}}{\mathrm{d} \mathbb{P}_{[0, t]}^{\mu,-}} \\
& =\frac{1}{t}\left[\int_{0}^{t}\left(Q^{\frac{1}{2}} F\left(X_{s}\right)\right)^{\prime} \mathrm{d} B_{s}+\frac{1}{2} \int_{0}^{t}\left|Q^{\frac{1}{2}} F\left(X_{s}\right)\right|^{2} \mathrm{~d} s\right] \\
& =\frac{1}{t}\left[\int_{0}^{t}\left(Q^{-\frac{1}{2}} N X_{s}\right)^{\prime} \mathrm{d} B_{s}+\frac{1}{2} \int_{0}^{t}\left|Q^{-\frac{1}{2}} N X_{s}\right|^{2} \mathrm{~d} s\right]
\end{aligned}
$$

where

$$
F(x)=2 Q^{-1} A x-\nabla \log \rho(x)=\left(2 Q^{-1} A+\Gamma^{-1}\right) x=Q^{-1} N x .
$$

Recall that the process $\left(X_{t}^{\mu}\right)_{t \geq 0}$ is reversible if $\mathbb{P}_{[s, t]}^{\mu}=\mathbb{P}_{[s, t]}^{\mu,-}$ for any $0 \leq s<t<\infty$. For such a process the sample EPR is simply zero. Thus we will only be concerned with situations where $X^{\mu}$ is not reversible. It is well known that the stationary Ornstein-Uhlenbeck process $\left(X_{t}^{\mu}\right)_{t \geq 0}$ is reversible if and only if the coefficients $A$ and $Q$ satisfy the symmetry condition $Q^{-1 / 2} A=\left(Q^{-1 / 2} A\right)^{\prime}$ (cf. [37, p. 1338]). From (1.5) it then follows that if $A$ is symmetric 
then, under our assumptions, the Markov process $X^{\mu}$ is reversible. Thus we will assume that $A$ is not symmetric. Together with the fact that $A$ is a normal matrix, this implies that not all of the eigenvalues of $A$ are real.

By the ergodic theorem, we have that,

$$
\text { as } t \rightarrow \infty, e_{p}(t) \text { converges a.s. to } \frac{1}{2} \int_{\mathbb{R}^{d}}\left|Q^{-\frac{1}{2}} N x\right|^{2} \rho(x) \mathrm{d} x \text {. }
$$

Our main result gives asymptotics of probabilities of deviations from the above law of large numbers limit by establishing the following large deviation principle.

Theorem 2.1 (Large Deviation Principle). Suppose that Assumption (A) and (1.2) are satisfied and that $A$ is not a symmetric matrix. Then the sample EPR $e_{p}(t)$ satisfies an LDP in $\mathbb{R}$ with the rate function I given as

$$
I(x)= \begin{cases}x \frac{\sqrt{1+\ell_{0}(x)}-1}{2}+\frac{1}{2} \sum_{k=1}^{d}\left(\sqrt{\alpha_{k}^{2}-\beta_{k}^{2} \ell_{0}(x)}+\alpha_{k}\right), & x \geq 0, \\ -x \frac{\sqrt{1+\ell_{0}(x)}+1}{2}+\frac{1}{2} \sum_{k=1}^{d}\left(\sqrt{\alpha_{k}^{2}-\beta_{k}^{2} \ell_{0}(x)}+\alpha_{k}\right), & x<0,\end{cases}
$$

where $\alpha_{k} \pm \mathrm{i} \beta_{k}$ are the eigenvalues of $A$, and $\ell_{0}(x)$ is the unique solution of the equation

$$
|x|=\sqrt{1+\ell} \times \sum_{k=1}^{d} \frac{\beta_{k}^{2}}{\sqrt{\alpha_{k}^{2}-\ell \beta_{k}^{2}}}, \quad-1 \leq \ell<\min _{k=1, \ldots, d}\left\{\frac{\alpha_{k}^{2}}{\beta_{k}^{2}}\right\},
$$

namely the following properties hold

(i) I has compact level sets, i.e. for every $K<\infty,\{x \in \mathbb{R}: I(x) \leq K\}$ is a compact set.

(ii) For each closed subset $F \subset \mathbb{R}$,

$$
\limsup _{t \rightarrow \infty} \frac{1}{t} \log \mathbb{P}^{\mu}\left(e_{p}(t) \in F\right) \leq-\inf _{x \in F} I(x) ;
$$

(iii) For each open subset $G \subset \mathbb{R}$,

$$
\liminf _{t \rightarrow \infty} \frac{1}{t} \log \mathbb{P}^{\mu}\left(e_{p}(t) \in G\right) \geq-\inf _{x \in G} I(x),
$$

where $\mathbb{P}^{\mu}$ is the measure under which $X_{0}$ is distributed according to $\mu$.

Remark 2.2. From (2.4) it follows that $\min _{k=1, \ldots, d}\left\{\frac{\alpha_{k}^{2}}{\beta_{k}^{2}}\right\}<\infty$. The uniqueness of solutions of (2.7) is argued in the proof of Theorem 2.1 given in Section 5.2.

In order to prove Theorem 2.1 we will apply Gärtner-Ellis Theorem (cf. [5]). For this we will need to compute the Cramér function of $e_{p}(t)$. Define, for $t \geq 0$ and $\lambda \in \mathbb{R}$,

$$
\begin{aligned}
\Lambda_{t}(\lambda) & :=\frac{1}{t} \log \mathbb{E}^{\mu} \exp \left\{t \lambda e_{p}(t)\right\} \\
& =\frac{1}{t} \log \mathbb{E}^{\mu} \exp \left\{\lambda \int_{0}^{t}\left(Q^{-\frac{1}{2}} N X_{s}\right)^{\prime} \mathrm{d} B_{s}+\frac{\lambda}{2} \int_{0}^{t}\left|Q^{-\frac{1}{2}} N X_{s}\right|^{2} \mathrm{~d} s\right\},
\end{aligned}
$$

where $\mathbb{E}^{\mu}$ is the expectation function associated with $\mathbb{P}^{\mu}$. For $\lambda \in \mathbb{R}$, define

$$
\Lambda(\lambda):=\lim _{t \rightarrow \infty} \Lambda_{t}(\lambda) \text { provided } \lim _{t \rightarrow \infty} \Lambda_{t}(\lambda) \text { exists }
$$

and

$$
\mathcal{D}_{\Lambda}:=\{\lambda \in \mathbb{R}: \Lambda(\lambda)<\infty\} .
$$

$\Lambda$ is called the Cramér function [5] associated with large deviations of $e_{p}(t)$. 
The following theorem gives a closed form expression for $\Lambda$ and its domain and also gives our main fluctuation theorem.

Theorem 2.3 (Fluctuation Theorem). Assume the same conditions as in Theorem 2.1. Let $\mathcal{D}$ be the closed interval given as

$$
\left[-\frac{1}{2}-\frac{1}{2} \sqrt{1+\min _{k=1, \ldots, d}\left\{\frac{\alpha_{k}^{2}}{\beta_{k}^{2}}\right\}},-\frac{1}{2}+\frac{1}{2} \sqrt{1+\min _{k=1, \ldots, d}\left\{\frac{\alpha_{k}^{2}}{\beta_{k}^{2}}\right\}}\right] .
$$

Then, we have

$$
\Lambda(\lambda)= \begin{cases}-\frac{1}{2} \sum_{k=1}^{d}\left(\sqrt{\alpha_{k}^{2}-4 \lambda(1+\lambda) \beta_{k}^{2}}+\alpha_{k}\right) & \lambda \in \mathcal{D}, \\ \infty, & \lambda \notin \mathcal{D},\end{cases}
$$

in particular, $\mathcal{D}_{\Lambda}=\mathcal{D}$. Furthermore, $\Lambda$ and I satisfy the following Cohen-Gallavotti symmetry properties:

$$
\Lambda(x)=\Lambda(-(1+x)), \quad I(x)=I(-x)-x, \text { for all } x \in \mathbb{R}
$$

We note that the results in [4] follow as a special case of Theorems 2.1 and 2.3. Also, as noted in the Introduction, under our assumptions, the Cramér function and the rate function for the LDP of $e_{p}(t)$ do not depend on the matrix $Q$ and since, formally speaking, the deterministic system with $Q=0$ has zero EPR, the model exhibits a phase transition in that the EPR changes discontinuously at $Q=0$.

2.2. An auxiliary SDE. From the definition of sample EPR in (2.2), and noting from (1.5) that $Q^{-\frac{1}{2}} N=N Q^{-\frac{1}{2}}$, we see that

$$
\begin{aligned}
e_{p}(t) & =\frac{1}{t}\left[\int_{0}^{t}\left(N Q^{-\frac{1}{2}} X_{s}\right)^{\prime} \mathrm{d} B_{s}+\frac{1}{2} \int_{0}^{t}\left|N Q^{-\frac{1}{2}} X_{s}\right|^{2} \mathrm{~d} s\right] \\
& =\frac{1}{t}\left[\int_{0}^{t}\left(N \hat{X}_{s}\right)^{\prime} \mathrm{d} B_{s}+\frac{1}{2} \int_{0}^{t}\left|N \hat{X}_{s}\right|^{2} \mathrm{~d} s\right]
\end{aligned}
$$

where $\hat{X}_{s}=Q^{-\frac{1}{2}} X_{s}$. Observe that $\hat{X}_{t}$ satisfies the equation

$$
\mathrm{d} \hat{X}_{t}=A \hat{X}_{t} \mathrm{~d} t+\mathrm{d} B_{t},
$$

and the Markov process $\hat{X}_{t}$ admits a unique invariant measure $\hat{\mu}$ with a density function

$$
\begin{aligned}
\hat{\rho}(x) & =(2 \pi)^{-\frac{d}{2}}(\operatorname{det}(\hat{\Gamma}))^{-\frac{1}{2}} \exp \left(-\frac{x^{\prime} \hat{\Gamma}^{-1} x}{2}\right) \\
& =(2 \pi)^{-\frac{d}{2}}|\operatorname{det}(M)|^{\frac{1}{2}} \exp \left(\frac{x^{\prime} M x}{2}\right),
\end{aligned}
$$

where $\hat{\Gamma}=\int_{0}^{\infty} e^{A s} e^{A^{\prime} s} \mathrm{~d} s=-M^{-1}$. Recalling the definition of $\Lambda_{t}(\lambda)$ from (2.8), we have

$$
\Lambda_{t}(\lambda)=\frac{1}{t} \log \mathbb{E}^{\hat{\mu}} \exp \left\{\lambda \int_{0}^{t}\left(N \hat{X}_{s}\right)^{\prime} \mathrm{d} B_{s}+\frac{\lambda}{2} \int_{0}^{t}\left|N \hat{X}_{s}\right|^{2} \mathrm{~d} s\right\} .
$$

From the above observation we see that, under our assumptions, the quantity $\Lambda_{t}(\lambda)$ and the distribution of $e_{p}(t)$ under the stationary distribution of $X$ are independent of the choice of $Q$. This fact explains why the rate function in the LDP and the Cramér's function in the FT do not 
depend on $Q$. In view of this invariance, in rest of this work we assume that $Q=\operatorname{Id}$, i.e. the identity matrix, and, instead of (1.1), consider the equation

$$
\mathrm{d} X_{t}=A X_{t} \mathrm{~d} t+\mathrm{d} B_{t} .
$$

Note that with the new definition, the invariant measure $\mu$ of $X$ is the measure $\hat{\mu}$ defined above.

In order to compute the Cramér function associated with $e_{p}(t)$, we introduce the following auxiliary equation. For $\lambda \in \mathbb{R}$, let $Y_{\lambda, t}$ solve the equation

$$
\mathrm{d} Y_{\lambda, t}=D_{\lambda} Y_{\lambda, t} \mathrm{~d} t+\mathrm{d} W_{t}
$$

where

$$
D_{\lambda}=A+\lambda N, \quad \lambda \in \mathbb{R} .
$$

By (1.5), it is clear that $D_{\lambda} D_{\lambda}^{\prime}=D_{\lambda}^{\prime} D_{\lambda}$ and

$$
M=D_{\lambda}+D_{\lambda}^{\prime}, \quad \Gamma_{\lambda}:=\int_{0}^{\infty} e^{D_{\lambda} s} e^{D_{\lambda}^{\prime} s} \mathrm{~d} s=-M^{-1} .
$$

Thus for every $\lambda$, the Markov process $\left(Y_{\lambda, t}\right)_{t \geq 0}$ has the same unique ergodic measure $\mu$ as that of $\left(X_{t}\right)_{t \geq 0}$ (cf. [37]). For $\lambda \in \mathbb{R}$, define

$$
\tilde{\Lambda}_{t}(\lambda)=\frac{1}{t} \log \mathbb{E}^{\mu} \exp \left\{\frac{1}{2} \lambda(1+\lambda) \int_{0}^{t}\left|Z_{\lambda, s}\right|^{2} \mathrm{~d} s\right\},
$$

where

$$
Z_{\lambda, s}=N Y_{\lambda, s}
$$

and $\mathbb{E}^{\mu}$ denotes the expectation with respect to the probability measure under which $Y_{\lambda, 0}$ is distributed as $\mu$. For $\lambda \in \mathbb{R}$, define

$$
\tilde{\Lambda}(\lambda):=\lim _{t \rightarrow \infty} \tilde{\Lambda}_{t}(\lambda) \text { provided } \lim _{t \rightarrow \infty} \tilde{\Lambda}_{t}(\lambda) \text { exists, }
$$

and

$$
\mathcal{D}_{\tilde{\Lambda}}:=\{\lambda \in \mathbb{R}: \tilde{\Lambda}(\lambda)<\infty\} .
$$

We shall show in Proposition 5.5 that

$$
\mathcal{D}_{\tilde{\Lambda}}=\mathcal{D}_{\Lambda}, \quad \tilde{\Lambda}=\Lambda .
$$

\section{The ANALysis of $\int_{0}^{T}\left|Z_{\lambda, s}\right|^{2} \mathrm{~d} s$}

In this section we will decompose $\int_{0}^{T}\left|Z_{\lambda, s}\right|^{2} \mathrm{~d} s$ into its Wiener-Itô chaos expansion and compute its exponential moments by analyzing an operator associated with its second order chaos. For a function $\psi \in L^{2}\left([0, T]^{2} ; \mathbb{R}^{d \times d}\right)$, we write $\int_{[0, T]^{2}} d W_{u_{1}}^{\prime} \psi\left(u_{1}, u_{2}\right) d W_{u_{2}}$ for the $\mathbb{R}$ valued random variable defined as

$$
\sum_{i, j=1}^{d} \int_{0}^{T}\left(\int_{0}^{t} \psi_{i j}(s, t) d W_{s}^{i}\right) d W_{t}^{j}+\sum_{i, j=1}^{d} \int_{0}^{T}\left(\int_{0}^{s} \psi_{i j}(s, t) d W_{t}^{j}\right) d W_{s}^{i} .
$$

Stochastic integrals of the form

$$
\int_{0}^{u_{1}} d W_{u_{1}}^{\prime} \psi\left(u_{1}, u_{2}\right), \int_{0}^{u_{2}} \psi\left(u_{1}, u_{2}\right) d W_{u_{2}}
$$

are $\mathbb{R}^{d}$ valued random variables interpreted in a similar manner. 
Proposition 3.1. Let, for $\lambda \in \mathbb{R}, Y_{\lambda, t}$ and $Z_{\lambda, t}$ be as defined in (2.14) and (2.17) respectively. Suppose that for some $x \in \mathbb{R}^{d}, Y_{\lambda, 0}=x$. Then, for all $T \geq 0$, we have

$$
\int_{0}^{T}\left|Z_{\lambda, s}\right|^{2} \mathrm{~d} s=S_{0}^{x}(T)+S_{\lambda, 1}^{x}(T)+S_{\lambda, 2}(T)
$$

where

$$
\begin{gathered}
S_{0}^{x}(T)=\left|\left(\int_{0}^{T} e^{s M} \mathrm{~d} s\right)^{\frac{1}{2}} N x\right|^{2}+\int_{0}^{T} \operatorname{tr}\left[N^{\prime} e^{u M} N\right](T-u) \mathrm{d} u \\
S_{\lambda, 1}^{x}(T)=\int_{0}^{T}\left(G_{\lambda, T}^{x}(u)\right)^{\prime} \mathrm{d} W_{u}, \quad S_{\lambda, 2}(T)=\frac{1}{2} \int_{[0, T]^{2}} \mathrm{~d} W_{u_{1}}^{\prime} H_{\lambda, T}\left(u_{1}, u_{2}\right) \mathrm{d} W_{u_{2}},
\end{gathered}
$$

with

$$
\begin{gathered}
G_{\lambda, T}^{x}(u)=2 N^{\prime} e^{-u D_{\lambda}^{\prime}}\left(\int_{u}^{T} e^{M s} \mathrm{~d} s\right) N x \\
H_{\lambda, T}\left(u_{1}, u_{2}\right)=2\left(e^{-u_{1} D_{\lambda}} N\right)^{\prime}\left(\int_{u_{1} \vee u_{2}}^{T} e^{M t} \mathrm{~d} t\right) e^{-u_{2} D_{\lambda}} N .
\end{gathered}
$$

Proof. From (2.14), for all $s \geq 0$,

$$
Y_{\lambda, s}=e^{s D_{\lambda}} x+\int_{0}^{s} e^{(s-u) D_{\lambda}} \mathrm{d} W_{u}
$$

which implies that

$$
Z_{\lambda, s}=N Y_{\lambda, s}=N e^{s D_{\lambda}} x+\int_{0}^{s} e^{(s-u) D_{\lambda}} N \mathrm{~d} W_{u} .
$$

where we have used $N D_{\lambda}=D_{\lambda} N$ (which is a consequence of (1.5)). Thus,

$$
\left|Z_{\lambda, s}\right|^{2}=\left|N e^{s D_{\lambda}} x\right|^{2}+2\left(N e^{s D_{\lambda}} x\right)^{\prime} \int_{0}^{s} e^{(s-u) D_{\lambda}} N \mathrm{~d} W_{u}+\left|\int_{0}^{s} e^{(s-u) D_{\lambda}} N \mathrm{~d} W_{u}\right|^{2} .
$$

Let us first consider the last term on the right hand side of (3.3). By (1.5) the matrices $N, D_{\lambda}$ and $D_{\lambda}^{\prime}$ commute and thus recalling that $D_{\lambda}^{\prime}+D_{\lambda}=M$, we have

$$
\begin{aligned}
\left|\int_{0}^{s} e^{(s-u) D_{\lambda}} N \mathrm{~d} W_{u}\right|^{2} & =\left(\int_{0}^{s} e^{-u D_{\lambda}} N \mathrm{~d} W_{u}\right)^{\prime} e^{s\left(D_{\lambda}^{\prime}+D_{\lambda}\right)}\left(\int_{0}^{s} e^{-u D_{\lambda}} N \mathrm{~d} W_{u}\right) \\
& =\left(\int_{0}^{s} e^{-u D_{\lambda}} N \mathrm{~d} W_{u}\right)^{\prime} e^{s M}\left(\int_{0}^{s} e^{-u D_{\lambda}} N \mathrm{~d} W_{u}\right) .
\end{aligned}
$$

For fixed $s$, applying Itô's formula to the semimartingale

$$
r \mapsto\left(\int_{0}^{r} e^{-u D_{\lambda}} N \mathrm{~d} W_{u}\right)^{\prime} e^{s M}\left(\int_{0}^{r} e^{-u D_{\lambda}} N \mathrm{~d} W_{u}\right),
$$

we have

$$
\begin{aligned}
& \left(\int_{0}^{r} e^{-u D_{\lambda}} N \mathrm{~d} W_{u}\right)^{\prime} e^{s M}\left(\int_{0}^{r} e^{-u D_{\lambda}} N \mathrm{~d} W_{u}\right) \\
= & 2 \int_{0}^{r}\left(\int_{0}^{u} e^{-v D_{\lambda}} N \mathrm{~d} W_{v}\right)^{\prime} e^{s M} e^{-u D_{\lambda}} N \mathrm{~d} W_{u}+\int_{0}^{r} \operatorname{tr}\left[N^{\prime} e^{-u D_{\lambda}^{\prime}} e^{s M} e^{-u D_{\lambda}} N\right] \mathrm{d} u \\
= & 2 \int_{0}^{r}\left(\int_{0}^{u} e^{-v D_{\lambda}} N \mathrm{~d} W_{v}\right)^{\prime} e^{s M} e^{-u D_{\lambda}} N \mathrm{~d} W_{u}+\int_{0}^{r} \operatorname{tr}\left[N^{\prime} e^{(s-u) M} N\right] \mathrm{d} u,
\end{aligned}
$$


where the last equality is once more from the fact that the matrices $N, D_{\lambda}$ and $D_{\lambda}^{\prime}$ commute, and that $D_{\lambda}^{\prime}+D_{\lambda}=M$. Taking $r=s$ in the above expression yields

$$
\left|\int_{0}^{s} e^{(s-u) D_{\lambda}} N \mathrm{~d} W_{u}\right|^{2}=2 \int_{0}^{s}\left(\int_{0}^{u} e^{-v D_{\lambda}} N \mathrm{~d} W_{v}\right)^{\prime} e^{s M} e^{-u D_{\lambda}} N \mathrm{~d} W_{u}+\int_{0}^{s} \operatorname{tr}\left[N^{\prime} e^{(s-u) M} N\right] \mathrm{d} u .
$$

The integral of the first term on the right hand side of (3.4) can be written as

$$
\begin{aligned}
& 2 \int_{0}^{T} \int_{0}^{s}\left(\int_{0}^{u} e^{-v D_{\lambda}} N \mathrm{~d} W_{v}\right)^{\prime} e^{s M} e^{-u D_{\lambda}} N \mathrm{~d} W_{u} \mathrm{~d} s \\
= & \int_{0}^{T} \int_{0}^{s}\left(\int_{0}^{u} e^{-v D_{\lambda}} N \mathrm{~d} W_{v}\right)^{\prime} e^{s M} e^{-u D_{\lambda}} N \mathrm{~d} W_{u} \mathrm{~d} s \\
& +\int_{0}^{T} \int_{0}^{s}\left(\int_{0}^{v} e^{-u D_{\lambda}} N \mathrm{~d} W_{u}\right)^{\prime} e^{s M} e^{-v D_{\lambda}} N \mathrm{~d} W_{v} \mathrm{~d} s \\
= & \int_{0}^{T}\left(\int_{0}^{u} \mathrm{~d} W_{v}^{\prime} e^{-v D_{\lambda}^{\prime}} N^{\prime}\right)\left(\int_{u}^{T} e^{s M} \mathrm{~d} s\right) e^{-u D_{\lambda}} N \mathrm{~d} W_{u} \\
& +\int_{0}^{T} \mathrm{~d} W_{v}^{\prime}\left[\left(\int_{v}^{T} e^{s M} \mathrm{~d} s\right) e^{-v D_{\lambda}^{\prime}} N^{\prime}\left(\int_{0}^{v} e^{-u D_{\lambda}} N \mathrm{~d} W_{u}\right)\right] \\
= & \frac{1}{2} \int_{[0, T]^{2}} \mathrm{~d} W_{u_{1}}^{\prime} H_{\lambda, T}\left(u_{1}, u_{2}\right) \mathrm{d} W_{u_{2}},
\end{aligned}
$$

where the second equality is by the (stochastic) Fubini Theorem (cf. [25, Section 3.7]). The integral of the second term on the right side of (3.4) can be written as

$$
\int_{0}^{T} \int_{0}^{s} \operatorname{tr}\left[N^{\prime} e^{(s-u) M} N\right] \mathrm{d} u \mathrm{~d} s=\int_{0}^{T} \int_{0}^{s} \operatorname{tr}\left[N^{\prime} e^{u M} N\right] \mathrm{d} u \mathrm{~d} s=\int_{0}^{T} \operatorname{tr}\left[N^{\prime} e^{u M} N\right](T-u) \mathrm{d} u .
$$

Combining the above observations the integral of the left side of (3.4) is given as

$$
\int_{0}^{T}\left|\int_{0}^{s} e^{(s-u) D_{\lambda}} N \mathrm{~d} W_{u}\right|^{2} \mathrm{~d} s=\frac{1}{2} \int_{[0, T]^{2}} \mathrm{~d} W_{u_{1}}^{\prime} H_{\lambda, T}\left(u_{1}, u_{2}\right) \mathrm{d} W_{u_{2}}+\int_{0}^{T} \operatorname{tr}\left[N^{\prime} e^{u M} N\right](T-u) \mathrm{d} u .
$$

For the first two terms on the right hand of (3.3), integrating over $s \in[0, T]$, we have

$$
\begin{aligned}
\int_{0}^{T}\left|N e^{s D_{\lambda}} x\right|^{2} \mathrm{~d} s & =\int_{0}^{T} x e^{s D_{\lambda}^{\prime}} N^{\prime} N e^{s D_{\lambda}} x \mathrm{~d} s \\
& =\int_{0}^{T} x^{\prime} N^{\prime} e^{s M} N x \mathrm{~d} s=\left|\left(\int_{0}^{T} e^{s M} \mathrm{~d} s\right)^{\frac{1}{2}} N x\right|^{2}
\end{aligned}
$$

and

$$
\begin{aligned}
2 \int_{0}^{T}\left(N e^{s D_{\lambda}} x\right)^{\prime} \int_{0}^{s} e^{(s-u) D_{\lambda}} N \mathrm{~d} W_{u} \mathrm{~d} s & =2 \int_{0}^{T}(N x)^{\prime} e^{s M} \int_{0}^{s} e^{-u D_{\lambda}} N \mathrm{~d} W_{u} \mathrm{~d} s \\
& =2 \int_{0}^{T}(N x)^{\prime}\left(\int_{u}^{T} e^{M s} \mathrm{~d} s\right) e^{-u D_{\lambda}} N \mathrm{~d} W_{u} \\
& =2 \int_{0}^{T}\left[N^{\prime} e^{-u D_{\lambda}^{\prime}}\left(\int_{u}^{T} e^{M s} \mathrm{~d} s\right) N x\right]^{\prime} \mathrm{d} W_{u},
\end{aligned}
$$


where the second equality once more uses the (stochastic) Fubini theorem. Combining the previous three relations with (3.3), we immediately get the desired identity in the proposition.

Let $T>0$ and $\lambda \in \mathbb{R}$. Recall the function $H_{\lambda, T}:[0, T] \times[0, T] \rightarrow \mathbb{R}^{d \times d}$ defined in Proposition 3.1. Define the operator $K_{\lambda, T}: L^{2}\left([0, T] ; \mathbb{R}^{d}\right) \rightarrow L^{2}\left([0, T] ; \mathbb{R}^{d}\right)$ by

$$
K_{\lambda, T} f(t)=\int_{0}^{T} H_{\lambda, T}(t, s) f(s) \mathrm{d} s, \quad f \in L^{2}\left([0, T] ; \mathbb{R}^{d}\right),
$$

Lemma 3.2. $K_{\lambda, T}$ is a nonnegative, symmetric, trace class operator on $L^{2}\left([0, T] ; \mathbb{R}^{d}\right)$. Consequently there exist a standard orthonormal basis $\left\{e_{n}\right\}_{n \geq 1}$ of $L^{2}\left([0, T] ; \mathbb{R}^{d}\right)$ and a real sequence (possibly depending on $\lambda$ and $T$ ) $\gamma_{1} \geq \gamma_{2} \geq \ldots \geq \gamma_{n} \geq \ldots \geq 0$, such that

$$
\begin{aligned}
& H_{\lambda, T}(s, t)=\sum_{n \geq 1} \gamma_{n} e_{n}(s) \otimes e_{n}(t), \quad 0 \leq s, t \leq T, \\
& K_{\lambda, T} e_{n}=\gamma_{n} e_{n}, \quad n=1,2, \ldots,
\end{aligned}
$$

where for $a, b \in \mathbb{R}^{d}$, a $\otimes b$ denotes a $d \times d$ matrix whose $(i, j)$-th entry is $a_{i} b_{j}$. Moreover, we have

$$
\begin{aligned}
\operatorname{tr}\left(K_{\lambda, T}\right) & =\int_{0}^{T} \operatorname{tr}\left(H_{\lambda, T}(u, u)\right) \mathrm{d} u \\
& =2\left[\operatorname{tr}\left(N^{\prime} M^{-1}\left(e^{M T}-\mathrm{Id}\right) M^{-1} N\right)-T \operatorname{tr}\left(N^{\prime} M^{-1} N\right)\right] .
\end{aligned}
$$

Proof. The symmetry of $K_{\lambda, T}$ is immediate from the symmetry of the function $H_{\lambda, T}$ (namely the property $H_{\lambda, T}(s, t)=H_{\lambda, T}(t, s)$ for $\left.(s, t) \in[0, T]^{2}\right)$. Now we show that $K_{\lambda, T}$ is nonnegative, i.e. for any $f \in L^{2}\left([0, T] ; \mathbb{R}^{d}\right)$, the following relation holds:

$$
\int_{[0, T]^{2}}\left(f\left(u_{1}\right)\right)^{\prime} H_{\lambda, T}\left(u_{1}, u_{2}\right) f\left(u_{2}\right) \mathrm{d} u_{1} \mathrm{~d} u_{2} \geq 0 .
$$

Since $M$ is a symmetric matrix, there exists some orthogonal matrix $P$ such that

$$
P M P^{\prime}=\operatorname{diag}\left\{\eta_{1}, \ldots, \eta_{d}\right\}=\operatorname{diag}(\boldsymbol{\eta}) \text { with } \eta_{i} \in \mathbb{R} \text { for } 1 \leq i \leq d .
$$

Therefore, for any $f \in L^{2}\left([0, T] ; \mathbb{R}^{d}\right)$, denote $g(t)=P e^{-t D_{\lambda}} N f(t)$, we have

$$
\begin{aligned}
\int_{[0, T]^{2}}\left(f\left(u_{1}\right)\right)^{\prime} H_{\lambda, T}\left(u_{1}, u_{2}\right) f\left(u_{2}\right) \mathrm{d} u_{1} \mathrm{~d} u_{2} & =2 \int_{[0, T]^{2}}\left(g\left(u_{1}\right)\right)^{\prime}\left(\int_{u_{1} \vee u_{2}}^{T} e^{\operatorname{diag}(\boldsymbol{\eta}) t} \mathrm{~d} t\right) g\left(u_{2}\right) \mathrm{d} u_{1} \mathrm{~d} u_{2} \\
& =2 \sum_{i=1}^{d} \int_{[0, T]^{2}} g_{i}\left(u_{1}\right)\left(\int_{u_{1} \vee u_{2}}^{T} e^{\eta_{i} t} \mathrm{~d} t\right) g_{i}\left(u_{2}\right) \mathrm{d} u_{1} \mathrm{~d} u_{2},
\end{aligned}
$$

where $g_{i}(t)$ is the $i$-th element of $g(t)$. For $1 \leq i \leq d$,

$$
\begin{aligned}
& \int_{[0, T]^{2}} g_{i}\left(u_{1}\right)\left(\int_{u_{1} \vee u_{2}}^{T} e^{\eta_{i} t} \mathrm{~d} t\right) g_{i}\left(u_{2}\right) \mathrm{d} u_{1} \mathrm{~d} u_{2} \\
= & 2 \int_{0}^{T} \int_{0}^{u_{2}} g_{i}\left(u_{1}\right) \mathrm{d} u_{1}\left(\int_{u_{2}}^{T} e^{\eta_{i} t} \mathrm{~d} t\right) g_{i}\left(u_{2}\right) \mathrm{d} u_{2} \\
= & \frac{2}{\eta_{i}}\left[\int_{0}^{T} \int_{0}^{u_{2}} g_{i}\left(u_{1}\right) \mathrm{d} u_{1} e^{\eta_{i} T} g_{i}\left(u_{2}\right) \mathrm{d} u_{2}-\int_{0}^{T} \int_{0}^{u_{2}} g_{i}\left(u_{1}\right) \mathrm{d} u_{1} e^{\eta_{i} u_{2}} g_{i}\left(u_{2}\right) \mathrm{d} u_{2}\right]
\end{aligned}
$$


Letting $F_{i}(t)=\int_{0}^{t} g_{i}(s) \mathrm{d} s$, by a straightforward calculation, we get

$$
\begin{aligned}
& \int_{0}^{T} \int_{0}^{u_{2}} g_{i}\left(u_{1}\right) \mathrm{d} u_{1} e^{\eta_{i} T} g_{i}\left(u_{2}\right) \mathrm{d} u_{2}=\frac{1}{2} e^{\eta_{i} T} F_{i}^{2}(T), \\
& \int_{0}^{T} \int_{0}^{u_{2}} g_{i}\left(u_{1}\right) \mathrm{d} u_{1} e^{\eta_{i} u_{2}} g_{i}\left(u_{2}\right) \mathrm{d} u_{2}=\frac{1}{2} e^{\eta_{i} T} F_{i}^{2}(T)-\frac{\eta_{i}}{2} \int_{0}^{T} e^{\eta_{i} t} F_{i}^{2}(t) \mathrm{d} t,
\end{aligned}
$$

thus,

$$
\int_{[0, T]^{2}} g_{i}\left(u_{1}\right)\left(\int_{u_{1} \vee u_{2}}^{T} e^{\eta_{i} t} \mathrm{~d} t\right) g_{i}\left(u_{2}\right) \mathrm{d} u_{1} \mathrm{~d} u_{2}=\int_{0}^{T} e^{\eta_{i} t} F_{i}^{2}(t) \mathrm{d} t .
$$

Using the above identities in (3.9), we have the desired inequality in (3.8).

Since $K_{\lambda, T}$ is a nonnegative symmetric operator, by Mercer's Theorem [14, Theorem 16.7.1], there exist a complete orthonormal system $\left\{e_{n}\right\}_{n \geq 1}$ in $L^{2}\left([0, T] ; \mathbb{R}^{d}\right)$ and a sequence of nonnegative reals $\left\{\gamma_{n}\right\}_{n \geq 1}$ such that $\gamma_{1} \geq \gamma_{2} \geq \ldots$ and that (3.6) holds. Moreover,

$$
\begin{aligned}
\int_{0}^{T} \operatorname{tr}\left(H_{\lambda, T}(u, u)\right) \mathrm{d} u & =2 \int_{0}^{T} \operatorname{tr}\left[N^{\prime} e^{-u D_{\lambda}^{\prime}}\left(\int_{u}^{T} e^{M t} \mathrm{~d} t\right) e^{-u D_{\lambda}} N\right] \mathrm{d} u \\
& =2 \int_{0}^{T} \operatorname{tr}\left[N^{\prime} e^{-u M}\left(\int_{u}^{T} e^{M t} \mathrm{~d} t\right) N\right] \mathrm{d} u \\
& =2 \int_{0}^{T} \operatorname{tr}\left[N^{\prime} M^{-1}\left(e^{M(T-u)}-\mathrm{Id}\right) N\right] \mathrm{d} u \\
& =2\left[\operatorname{tr}\left(N^{\prime} M^{-1}\left(e^{M T}-\mathrm{Id}\right) M^{-1} N\right)-T \operatorname{tr}\left(N^{\prime} M^{-1} N\right)\right],
\end{aligned}
$$

where the second equality is by using the the commuting property of matrices $M, D_{\lambda}$ and $D_{\lambda}^{\prime}$, and the fact that $D_{\lambda}+D_{\lambda}^{\prime}=M$. This shows that $K_{\lambda, T}$ is a trace class operator and that $\operatorname{tr}\left(K_{\lambda, T}\right)$ is given by (3.7).

Lemma 3.2 shows that $K_{\lambda, T}$ is a symmetric compact (in fact trace class) operator with the spectrum $\sigma\left(K_{\lambda, T}\right)$ given as

$$
\sigma\left(K_{\lambda, T}\right)=\left\{\gamma_{1}, \gamma_{2}, \ldots\right\}
$$

and $\operatorname{tr}\left(K_{\lambda, T}\right)=\sum_{i=1}^{\infty} \gamma_{i}<\infty$.

Theorem 3.3. Let, for $\lambda \in \mathbb{R}, Y_{\lambda, t}$ and $Z_{\lambda, t}$ be as defined in (2.14) and (2.17) respectively. Suppose that for some $x \in \mathbb{R}^{d}, Y_{\lambda, 0}=x$. Then for any $\theta \in\left(-\infty, \frac{1}{\gamma_{1}}\right)$, where $\gamma_{i}$ are as in Lemma 3.2, we have

$$
\begin{aligned}
\mathbb{E}^{x} & \exp \left(\theta \int_{0}^{T}\left|Z_{\lambda, s}\right|^{2} \mathrm{~d} s\right) \\
= & \frac{1}{\sqrt{\operatorname{det}\left(\operatorname{Id}-\theta K_{\lambda, T}\right)}} \\
& \times \exp \left[\theta S_{0}^{x}(T)-\frac{\theta}{2} \operatorname{tr}\left(K_{\lambda, T}\right)+\frac{\theta^{2}}{2}\left\langle G_{\lambda, T}^{x},\left(\operatorname{Id}-\theta K_{\lambda, T}\right)^{-1} G_{\lambda, T}^{x}\right\rangle_{L^{2}\left([0, T] ; \mathbb{R}^{d}\right)}\right],
\end{aligned}
$$

where $S_{0}^{x}$ and $G_{\lambda, T}^{x}$ are as in Proposition 3.1 and

$$
\frac{1}{\sqrt{\operatorname{det}\left(\mathrm{Id}-\theta K_{\lambda, T}\right)}}=\prod_{n=1}^{\infty} \frac{1}{\sqrt{1-\theta \gamma_{n}}} .
$$


Moreover, for all $\theta \geq \frac{1}{\gamma_{1}}$, we have

$$
\mathbb{E}^{x} \exp \left(\theta \int_{0}^{T}\left|Z_{\lambda, s}\right|^{2} \mathrm{~d} s\right)=\infty
$$

Proof. For notational simplicity, we drop the subscript $x$ in $\mathbb{E}^{x}$. Denote

$$
W\left(e_{n}\right)=\int_{0}^{T}\left(e_{n}(s)\right)^{\prime} \mathrm{d} W_{s},
$$

where $\left\{e_{n}\right\}$ is as in Lemma 3.2. It is easy to verify that

$$
\mathbb{E}\left[W\left(e_{n}\right) W\left(e_{m}\right)\right]=\int_{0}^{T}\left(e_{n}(s)\right)^{\prime} e_{n}(s) \mathrm{d} s=\delta_{m n}, \text { for } m, n \in \mathbb{N}
$$

and hence $W\left(e_{1}\right), W\left(e_{2}\right), \ldots$ is a sequence of i.i.d. standard normal random variables.

From (3.6), we have

$$
\int_{[0, T]^{2}} \mathrm{~d} W_{u_{1}}^{\prime} H_{\lambda, T}\left(u_{1}, u_{2}\right) \mathrm{d} W_{u_{2}}=\sum_{n=1}^{\infty} \gamma_{n} \int_{[0, T]^{2}} \mathrm{~d} W_{u_{1}}^{\prime} e_{n}\left(u_{1}\right) \otimes e_{n}\left(u_{2}\right) \mathrm{d} W_{u_{2}},
$$

where the series converges in $L^{2}\left(\mathbb{P}^{x}\right)$. Also by definition of the multiple Wiener-Itô integrals,

$$
\int_{[0, T]^{2}} \mathrm{~d} W_{u_{1}}^{\prime} e_{n}\left(u_{1}\right) \otimes e_{n}\left(u_{2}\right) \mathrm{d} W_{u_{2}}=2 \int_{0}^{T}\left(e_{n}\left(u_{1}\right)\right)^{\prime}\left(\int_{0}^{u_{1}}\left(e_{n}\left(u_{2}\right)\right)^{\prime} \mathrm{d} W_{u_{2}}\right) \mathrm{d} W_{u_{1}} .
$$

Applying Itô formula to $\left(\int_{0}^{t}\left(e_{n}(s)\right)^{\prime} \mathrm{d} W_{s}\right)^{2}$, we get

$$
\left(\int_{0}^{T}\left(e_{n}(s)\right)^{\prime} \mathrm{d} W_{s}\right)^{2}=2 \int_{0}^{T}\left(e_{n}\left(u_{1}\right)\right)^{\prime}\left(\int_{0}^{u_{1}}\left(e_{n}\left(u_{2}\right)\right)^{\prime} \mathrm{d} W_{u_{2}}\right) \mathrm{d} W_{u_{1}}+\int_{0}^{T}\left|e_{n}(s)\right|^{2} \mathrm{~d} s .
$$

This, together with the fact $\int_{0}^{T}\left|e_{n}(s)\right|^{2} \mathrm{~d} s=1$, immediately implies

$$
\int_{[0, T]^{2}} \mathrm{~d} W_{u_{1}}^{\prime} H_{\lambda, T}\left(u_{1}, u_{2}\right) \mathrm{d} W_{u_{2}}=\sum_{n=1}^{\infty} \gamma_{n}\left[\left(W\left(e_{n}\right)\right)^{2}-1\right] .
$$

In rest of the proof we suppress $x$ in the notation $S_{0}^{x}$ and $G_{\lambda, T}^{x}$. Since $G_{\lambda, T} \in L^{2}\left([0, T] ; \mathbb{R}^{d}\right)$, we can represent it as $G_{\lambda, T}(t)=\sum_{n=1}^{\infty} g_{\lambda, n} e_{n}(t)$ with the series converging in $L^{2}\left([0, T] ; \mathbb{R}^{d}\right)$ and $\left(g_{\lambda, n}\right)_{n \geq 1}$ being a sequence of real numbers with $\sum_{n} g_{\lambda, n}^{2}<\infty$. Henceforth, we suppress the subscript $\lambda$ in $g_{\lambda, n}$. Then

$$
\int_{0}^{T}\left(G_{\lambda, T}(t)\right)^{\prime} \mathrm{d} W_{t}=\sum_{n=1}^{\infty} g_{n} W\left(e_{n}\right) .
$$

Hence, combining the above two identities, we have from Proposition 3.1

$$
\int_{0}^{T}\left|Z_{\lambda, s}\right|^{2} \mathrm{~d} s=S_{0}^{x}(T)+\sum_{n=1}^{\infty} g_{n} W\left(e_{n}\right)+\frac{1}{2} \sum_{n=1}^{\infty} \gamma_{n}\left[\left(W\left(e_{n}\right)\right)^{2}-1\right] .
$$


Let $N \in \mathbb{N}$. Since $W\left(e_{1}\right), W\left(e_{2}\right), \ldots$ are i.i.d. standard normal random variables, if $\theta \gamma_{1}<1$ we have

$$
\begin{aligned}
& \mathbb{E} \exp \left(\theta \sum_{n=1}^{N} g_{n} W\left(e_{n}\right)+\frac{\theta}{2} \sum_{n=1}^{N} \gamma_{n}\left\{\left[W\left(e_{n}\right)\right]^{2}-1\right\}\right) \\
= & \exp \left(-\frac{\theta}{2} \sum_{n=1}^{N} \gamma_{n}\right) \prod_{n=1}^{N} \mathbb{E} \exp \left(\theta g_{n} W\left(e_{n}\right)+\frac{\theta \gamma_{n}}{2}\left[W\left(e_{n}\right)\right]^{2}\right) \\
= & {\left[\prod_{n=1}^{N} \frac{1}{\sqrt{1-\theta \gamma_{n}}}\right] \exp \left(-\frac{\theta}{2} \sum_{n=1}^{N} \gamma_{n}+\frac{\theta^{2}}{2} \sum_{n=1}^{N} \frac{g_{n}^{2}}{1-\theta \gamma_{n}}\right) . }
\end{aligned}
$$

Recall that $\sum_{n=1}^{\infty} \gamma_{n}<\infty$ and $\sum_{n=1}^{\infty} g_{n}^{2}<\infty$, whence

$$
\prod_{n=1}^{\infty} \frac{1}{\sqrt{1-\theta \gamma_{n}}}<\infty, \quad \sum_{n=1}^{N} \frac{g_{n}^{2}}{1-\theta \gamma_{n}} \leq \sum_{n=1}^{N} \frac{g_{n}^{2}}{1-\theta \gamma_{1}} \leq \sum_{n=1}^{\infty} \frac{g_{n}^{2}}{1-\theta \gamma_{1}}<\infty
$$

this implies

$$
\lim _{N \rightarrow \infty}\left[\prod_{n=1}^{N} \frac{1}{\sqrt{1-\theta \gamma_{n}}}\right] \exp \left(-\frac{\theta}{2} \sum_{n=1}^{N} \gamma_{n}+\frac{\theta^{2}}{2} \sum_{n=1}^{N} \frac{g_{n}^{2}}{1-\theta \gamma_{n}}\right) \text { exists. }
$$

Thus we have shown that, for all $\theta<1 / \gamma_{1}$,

$$
\begin{aligned}
& \lim _{N \rightarrow \infty} \mathbb{E} \exp \left(\theta \sum_{n=1}^{N} g_{n} W\left(e_{n}\right)+\frac{\theta}{2} \sum_{n=1}^{N} \gamma_{n}\left\{\left[W\left(e_{n}\right)\right]^{2}-1\right\}\right) \\
= & \frac{1}{\sqrt{\operatorname{det}\left(\operatorname{Id}-\theta K_{\lambda, T}\right)}} \exp \left(-\frac{\theta}{2} \operatorname{tr}\left(K_{\lambda, T}\right)+\frac{\theta^{2}}{2}\left\langle G_{\lambda, T},\left(\operatorname{Id}-\theta K_{\lambda, T}\right)^{-1} G_{\lambda, T}\right\rangle_{L^{2}\left([0, T] ; \mathbb{R}^{d}\right)}\right)
\end{aligned}
$$

where we have used the observation that

$$
\left\langle G_{\lambda, T},\left(\mathrm{Id}-\theta K_{\lambda, T}\right)^{-1} G_{\lambda, T}\right\rangle_{L^{2}\left([0, T] ; \mathbb{R}^{d}\right)}=\sum_{n=1}^{\infty} \frac{g_{n}^{2}}{1-\theta \gamma_{n}} .
$$

In order to complete the proof of the first statement in the theorem it suffices to show that in (3.15) the order of the limit $\lim _{N \rightarrow \infty}$ and $\mathbb{E}$ can be interchanged. For this it is enough to show that for all $\theta<1 / \gamma_{1}$

$$
\sup _{N \geq 1} \mathbb{E} \exp \left(\theta \sum_{n=1}^{N} g_{n} W\left(e_{n}\right)+\frac{\theta}{2} \sum_{n=1}^{N} \gamma_{n}\left\{\left[W\left(e_{n}\right)\right]^{2}-1\right\}\right)<\infty
$$

This follows on observing that the above expectation is bounded above by

$$
\frac{1}{\sqrt{\operatorname{det}\left(\operatorname{Id}-\theta K_{\lambda, T}\right)}} \exp \left(\frac{|\theta|}{2} \operatorname{tr}\left(K_{\lambda, T}\right)+\frac{\theta^{2}}{2} \sum_{n=1}^{\infty} \frac{g_{n}^{2}}{1-\theta \gamma_{n}}\right),
$$

which is clearly finite for every $\theta<\frac{1}{\gamma_{1}}$. This proves the first statement in the theorem. The second statement is an immediate consequence of the fact that for $\theta \geq 1 / \gamma_{1}, \mathbb{E} \exp \left\{\theta \gamma_{1}\left(W\left(e_{1}\right)\right)^{2}\right\}=$ $\infty$. 


\section{THE EIGENVALUES OF THE OPERATOR $K_{\lambda, T}$}

Recall from Lemma 3.2 that the operator $K_{\lambda, T}: L^{2}\left([0, T] ; \mathbb{R}^{d}\right) \rightarrow L^{2}\left([0, T] ; \mathbb{R}^{d}\right)$ is symmetric and has nonnegative eigenvalues $\gamma_{1} \geq \gamma_{2} \geq \ldots \geq 0$. This section is devoted to analyzing these eigenvalues. To this end, we extend the operator $K_{\lambda, T}$, in a natural fashion, from $L^{2}\left([0, T] ; \mathbb{R}^{d}\right)$ to $L^{2}\left([0, T] ; \mathbb{C}^{d}\right)$, namely the complex valued $L^{2}$ function space with inner product:

$$
\langle f, g\rangle_{L^{2}\left([0, T] ; \mathbb{C}^{d}\right)}=\int_{0}^{T} \sum_{i=1}^{d} f_{i}(t) \overline{g_{i}(t)} \mathrm{d} t, \quad f, g \in L^{2}\left([0, T] ; \mathbb{C}^{d}\right)
$$

Note that $K_{\lambda, T}$ is also a symmetric operator on $L^{2}\left([0, T] ; \mathbb{C}^{d}\right)$ and that $\left\{e_{n}\right\}_{n \geq 1}$ is also an orthonormal basis in $L^{2}\left([0, T] ; \mathbb{C}^{d}\right)$. This extension will allow us to use tools from complex function theory. For notational simplicity, from now on we denote

$$
\mathcal{H}=L^{2}\left([0, T] ; \mathbb{C}^{d}\right)
$$

For a complex matrix $V, V^{*}$ will denote its conjugate transpose. Note that when the matrix $V$ is real $V^{*}=V^{\prime}$.

Since $A$ is a normal matrix, there is a complex unitary matrix $U$ (cf. [18, Thmeorem 2.5.8]) such that

$$
U^{*} A U=\operatorname{diag}\left\{a_{1}, \ldots, a_{d}\right\}
$$

where $a_{i} \in \mathbb{C}$.

Write $U=\left[U_{1}, \ldots, U_{d}\right]$ with $U_{k}$ being the column vectors of $U$. These vectors form an orthonormal basis of $\mathbb{C}^{d}$. Denote by $P_{U_{k}}$ the orthogonal projection from $\mathbb{C}^{d}$ to the subspace spanned by the vector $U_{k}$, which can be represented as $P_{U_{k}}=U_{k} \otimes U_{k}^{*}$ under the basis $U_{1}, \ldots, U_{d}$. From this it is easily seen that $P_{U_{i}}=P_{U_{i}}^{*}$ for each $i, P_{U_{i}} P_{U_{j}}=0$ for $i \neq j$, and $\operatorname{Id}=\sum_{k=1}^{d} P_{U_{k}}$. It follows from the above spectrum calculation that, with $a_{k}=\alpha_{k}+\mathrm{i} \beta_{k}$,

$$
\begin{aligned}
& A=\sum_{k=1}^{d} a_{k} P_{U_{k}}, \quad A^{\prime}=A^{*}=\sum_{k=1}^{d} \bar{a}_{k} P_{U_{k}}, \\
& D_{\lambda}=\sum_{k=1}^{d}\left(a_{k}+\mathrm{i} 2 \lambda \beta_{k}\right) P_{U_{k}}, \quad D_{\lambda}^{*}=\sum_{k=1}^{d}\left(\bar{a}_{k}-\mathrm{i} 2 \lambda \beta_{k}\right) P_{U_{k}}, \\
& M=A+A^{*}=\sum_{k=1}^{d} 2 \alpha_{k} P_{U_{k}}, \quad N=A-A^{*}=\sum_{k=1}^{d} 2 \mathrm{i} \beta_{k} P_{U_{k}},
\end{aligned}
$$

and that

$$
\left|\left(\int_{0}^{T} e^{s M} \mathrm{~d} s\right)^{\frac{1}{2}} N x\right|^{2}=\sum_{k=1}^{d} \frac{2 \beta_{k}^{2}}{\alpha_{k}}\left(e^{\alpha_{k} T}-1\right)\left|\left\langle x, U_{k}\right\rangle_{\mathbb{C}^{d}}\right|^{2}
$$


and

$$
\begin{aligned}
G_{\lambda, T}^{x}(u) & =2 N^{\prime} e^{-u D_{\lambda}^{\prime}}\left(\int_{u}^{T} e^{M s} \mathrm{~d} s\right) N x \\
& =\sum_{k=1}^{d} \frac{4 \beta_{k}^{2}}{\alpha_{k}} e^{-\left(\bar{a}_{k}-\mathrm{i} 2 \lambda \beta_{k}\right) u}\left(e^{2 \alpha_{k} T}-e^{2 \alpha_{k} u}\right)\left\langle x, U_{k}\right\rangle_{\mathbb{C}^{d}} U_{k}, \\
H_{\lambda, T}\left(u_{1}, u_{2}\right) & =2\left(e^{-u_{1} D_{\lambda}} N\right)^{*}\left(\int_{u_{1} \vee u_{2}}^{T} e^{M t} \mathrm{~d} t\right) e^{-u_{2} D_{\lambda}} N \\
& =\sum_{k=1}^{d} \frac{-4 \beta_{k}^{2}}{\alpha_{k}} e^{-\left(\bar{a}_{k}-\mathrm{i} 2 \lambda \beta_{k}\right) u_{1}-\left(a_{k}+\mathrm{i} 2 \lambda \beta_{k}\right) u_{2}}\left(e^{2 \alpha_{k}\left(u_{1} \vee u_{2}\right)}-e^{2 \alpha_{k} T}\right) P_{U_{k}},
\end{aligned}
$$

where for $x, y \in \mathbb{C}^{d},\langle x, y\rangle_{\mathbb{C}^{d}}:=\sum_{i=1}^{d} x_{i} \bar{y}_{i}$. Let $\mathcal{H}_{k}=P_{U_{k}} \mathcal{H}$ where $P_{U_{k}} \mathcal{H}=\left\{P_{U_{k}} f: f \in \mathcal{H}\right\}$. Clearly $\mathcal{H}=\bigoplus_{i=1}^{d} \mathcal{H}_{k}$, i.e.,

$$
f=\oplus_{k=1}^{d} P_{U_{k}} f, \quad f \in \mathcal{H} .
$$

By (3.5) and (4.5), for any $f \in \mathcal{H}$, we have

$$
K_{\lambda, T} f\left(u_{1}\right)=\sum_{k=1}^{d} \frac{-4 \beta_{k}^{2}}{\alpha_{k}} \int_{0}^{T} e^{-\left(\bar{a}_{k}-\mathrm{i} 2 \lambda \beta_{k}\right) u_{1}-\left(a_{k}+\mathrm{i} 2 \lambda \beta_{k}\right) u_{2}}\left(e^{2 \alpha_{k}\left(u_{1} \vee u_{2}\right)}-e^{2 \alpha_{k} T}\right) P_{U_{k}} f\left(u_{2}\right) \mathrm{d} u_{2} .
$$

In particular, $K_{\lambda, T}$ maps $\mathcal{H}_{k}$ to itself. Let

$$
K_{\lambda, T}^{(k)}:=\left.K_{\lambda, T}\right|_{\mathcal{H}_{k}},
$$

be the restriction of the operator $K_{\lambda, T}$ to $\mathcal{H}_{k}$. For any $f \in \mathcal{H}_{k}$, which is isomorphic to a function in $L^{2}([0, T] ; \mathbb{C})$, we have

$$
K_{\lambda, T}^{(k)} f(t)=\frac{-4 \beta_{k}^{2}}{\alpha_{k}} \int_{0}^{T} e^{-\left(\bar{a}_{k}-\mathrm{i} 2 \lambda \beta_{k}\right) t-\left(a_{k}+\mathrm{i} 2 \lambda \beta_{k}\right) s}\left(e^{2 \alpha_{k}(t \vee s)}-e^{2 \alpha_{k} T}\right) f(s) \mathrm{d} s, \quad t \in[0, T] .
$$

Recall from (3.10) that $\sigma\left(K_{\lambda, T}\right)$ denotes the spectrum of $K_{\lambda, T}$. Denote by $\sigma\left(K_{\lambda, T}^{(k)}\right)$ the spectrum of $K_{\lambda, T}^{(k)}$. It is easy to see that

$$
\sigma\left(K_{\lambda, T}\right)=\bigcup_{k=1}^{d} \sigma\left(K_{\lambda, T}^{(k)}\right)=\left\{\gamma_{j}^{(k)}: j=1,2, \ldots, k=1, \ldots, d\right\},
$$

where some of $\gamma_{j}^{(k)}$ may take the same value. Indeed, the eigenvalues and eigenfunctions of $K_{\lambda, T}^{(k)}$ are clearly those of $K_{\lambda, T}$, which implies $\sigma\left(K_{\lambda, T}\right) \supset \bigcup_{k=1}^{d} \sigma\left(K_{\lambda, T}^{(k)}\right)$. On the other hand, if $\gamma$ and $f$ are an eigenvalue and the corresponding eigenfunction of $K_{\lambda, T}$, respectively (that is $K_{\lambda, T} f=\gamma f$ ), then by (4.6)-(4.8),

$$
\oplus_{k=1}^{d} K_{\lambda, T}^{(k)} P_{U_{k}} f=\oplus_{k=1}^{d} \gamma P_{U_{k}} f,
$$

from which it follows that $\gamma$ must be an eigenvalue of some $K_{\lambda, T}^{(k)}$. This shows that $\sigma\left(K_{\lambda, T}\right) \subset$ $\bigcup_{k=1}^{d} \sigma\left(K_{\lambda, T}^{(k)}\right)$.

The next proposition identifies a complex Sturm-Liouville problem governing the eigenfunctions of $K_{\lambda, T}^{(k)}$. This result will enable us to obtain useful bounds on the eigenvalues of this operator. 
Proposition 4.1. Let $1 \leq k \leq d$ be such that $\beta_{k} \neq 0$. Then $\operatorname{Ker}\left(K_{\lambda, T}^{(k)}\right)=\{0\}$. If $f \in \mathcal{H}_{k}$ is nonzero and $K_{\lambda, T}^{(k)} f=\gamma f$, then $f$ is a solution of the differential equation on $[0, T]$ :

$$
f^{\prime \prime}-2 \mathrm{i}(1+2 \lambda) \beta_{k} f^{\prime}-\left[(1+2 \lambda)^{2} \beta_{k}^{2}-\left(\frac{8 \beta_{k}^{2}}{\gamma}-\alpha_{k}^{2}\right)\right] f=0,
$$

subject to the separated boundary conditions

$$
\left\{\begin{array}{l}
{\left[\alpha_{k}-\mathrm{i}(1+2 \lambda) \beta_{k}\right] f(0)+f^{\prime}(0)=0} \\
f(T)=0
\end{array}\right.
$$

Proof. Let $\theta_{k}=a_{k}+2 \mathrm{i} \lambda \beta_{k}$ and for $f \in \mathcal{H}_{k}$ let $\tilde{K}_{\lambda, T}^{(k)} f=-\frac{\alpha_{k}}{4 \beta_{k}^{2}} K_{\lambda, T}^{(k)} f$. It follows from (4.9) that

$$
\begin{aligned}
\tilde{K}_{\lambda, T}^{(k)} f(t) & =e^{-\bar{\theta}_{k} t}\left(e^{2 \alpha_{k} t}-e^{2 \alpha_{k} T}\right) \int_{0}^{t} e^{-\theta_{k} s} f(s) \mathrm{d} s \\
& +e^{-\bar{\theta}_{k} t} \int_{t}^{T} e^{-\theta_{k} s}\left(e^{2 \alpha_{k} s}-e^{2 \alpha_{k} T}\right) f(s) \mathrm{d} s .
\end{aligned}
$$

Thus, $\tilde{K}_{\lambda, T}^{(k)} f(t), t \in[0, T]$ is absolutely continuous. By differentiating both sides of (4.13) with respect to $t$, we obtain

$$
\left(\tilde{K}_{\lambda, T}^{(k)} f\right)^{\prime}=-\bar{\theta}_{k}\left(\tilde{K}_{\lambda, T}^{(k)} f\right)+2 \alpha_{k} e^{\theta_{k} t} \int_{0}^{t} e^{-\theta_{k} s} f(s) \mathrm{d} s,
$$

which implies that

$$
2 \alpha_{k} e^{\theta_{k} t} \int_{0}^{t} e^{-\theta_{k} s} f(s) \mathrm{d} s=\left(\tilde{K}_{\lambda, T}^{(k)} f\right)^{\prime}+\bar{\theta}_{k}\left(\tilde{K}_{\lambda, T}^{(k)} f\right) .
$$

Similarly, $\left(\tilde{K}_{\lambda, T}^{(k)} f\right)^{\prime}(t), t \in[0, T]$ is absolutely continuous and we have that

$$
\left(\tilde{K}_{\lambda, T}^{(k)} f(t)\right)^{\prime \prime}=-\bar{\theta}_{k}\left(\tilde{K}_{\lambda, T}^{(k)} f(t)\right)^{\prime}+2 \alpha_{k} \theta_{k} e^{\theta_{k} t} \int_{0}^{t} e^{-\theta_{k} s} f(s) \mathrm{d} s+2 \alpha_{k} f(t) .
$$

Substituting (4.14) into (4.15), we have that

$$
\left(\tilde{K}_{\lambda, T}^{(k)} f\right)^{\prime \prime}=\left(\theta_{k}-\bar{\theta}_{k}\right)\left(\tilde{K}_{\lambda, T}^{(k)} f\right)^{\prime}+\left|\theta_{k}\right|^{2} \tilde{K}_{\lambda, T}^{(k)} f+2 \alpha_{k} f(t) .
$$

Since $\alpha_{k}<0$, if for $f \in \mathcal{H}_{k}, K_{\lambda, T}^{(k)} f=0$ we have from (4.16) immediately that $f(t) \equiv 0$. This shows that $\operatorname{Ker}\left(K_{\lambda, T}^{(k)}\right)=\{0\}$.

If instead $f \in \mathcal{H}_{k}$ is such that $K_{\lambda, T}^{(k)} f=\gamma f$ with $\gamma \neq 0$, then substituting $t=0$ in (4.14) and $t=T$ in (4.13) gives the separated boundary condition (4.12). Furthermore (4.16) implies that (4.11) holds.

Now we solve the Sturm-Liouville problem (4.11) and (4.12) for a fixed $k$ with $\beta_{k} \neq 0$, and provide estimates for the eigenvalues of $K_{\lambda, T}$. We consider three cases corresponding to different range of values of $\gamma$.

Case I: $\gamma>\frac{8 \beta_{k}^{2}}{\alpha_{k}^{2}}$. Let $v=\sqrt{\alpha_{k}^{2}-\frac{8 \beta_{k}^{2}}{\gamma}}$. Note that $v>0$. The general solution of equation (4.11) in this case is

$$
f(s)=e^{\mathrm{i}(1+2 \lambda) \beta_{k} s}\left(c_{1} e^{v s}+c_{2} e^{-v s}\right),
$$

where $c_{1}, c_{2}$ are constants. The boundary condition (4.12) gives

$$
\left\{\begin{array}{l}
\left(\alpha_{k}+v\right) c_{1}+\left(\alpha_{k}-v\right) c_{2}=0 \\
c_{1} e^{v T}+c_{2} e^{-v T}=0
\end{array}\right.
$$


It is easy to check that since $v>0$ and $\alpha_{k}<0$, we must have $c_{1}=c_{2}=0$ and thus $f=0$ is the only solution in this case.

Case II: $\gamma=\frac{8 \beta_{k}^{2}}{\alpha_{k}^{2}}$. The general solution of equation (4.11) in this case is

$$
f(s)=e^{\mathrm{i}(1+2 \lambda) \beta_{k} s}\left(c_{1}+c_{2} s\right),
$$

where $c_{1}, c_{2}$ are constants. The boundary condition (4.12) gives

$$
\left\{\begin{array}{l}
\alpha_{k} c_{1}+c_{2}=0 \\
c_{1}+T c_{2}=0
\end{array}\right.
$$

Since $\alpha_{k} T-1<0$, once more we must have $c_{1}=c_{2}=0$.

Case III: $\gamma<\frac{8 \beta_{k}^{2}}{\alpha_{k}^{2}}$. Let $\omega=\sqrt{\frac{8 \beta_{k}^{2}}{\gamma}-\alpha_{k}^{2}}$. Note that $\omega>0$. The general solution of equation (4.11) is

$$
f(s)=c_{1} e^{\mathrm{i}\left((1+2 \lambda) \beta_{k}+\omega\right) s}+c_{2} e^{\mathrm{i}\left((1+2 \lambda) \beta_{k}-\omega\right) s},
$$

where $c_{1}, c_{2}$ are constants. The boundary condition (4.12) gives

$$
\left\{\begin{array}{l}
\left(\alpha_{k}+\mathrm{i} \omega\right) c_{1}+\left(\alpha_{k}-\mathrm{i} \omega\right) c_{2}=0 \\
e^{\mathrm{i} \omega T} c_{1}+e^{-\mathrm{i} \omega T} c_{2}=0
\end{array}\right.
$$

In order to have constants $c_{1}$ and $c_{2}$ such that $c_{1}^{2}+c_{2}^{2} \neq 0$, we need

$$
\left(\alpha_{k}+\mathrm{i} \omega\right) e^{-\mathrm{i} \omega T}=\left(\alpha_{k}-\mathrm{i} \omega\right) e^{\mathrm{i} \omega T},
$$

which can be rewritten as

$$
\frac{\omega}{\alpha_{k}}=\tan w T
$$

Observe that $\tan w T$ is a periodic function with period $\frac{\pi}{T}$, whose restriction on $\left(-\frac{\pi}{2 T}, \frac{\pi}{2 T}\right)$ is a function crossing the origin and tending to $-\infty$ and $+\infty$ at $-\frac{\pi}{2 T}$ and $\frac{\pi}{2 T}$ respectively, and that $\frac{\omega}{\alpha_{k}}$ is a linear function crossing the origin and lying in the second and fourth quadrants. Thus there is a unique intersection point between $\frac{\omega}{\alpha_{k}}$ and $\tan w T$ in each period $\left[\frac{(2 j-1) \pi}{2 T}, \frac{(2 j+1) \pi}{2 T}\right]$ with $j \in \mathbb{Z}$. Since $\omega>0$, we only need to consider positive solutions to (4.19). Denoting these solutions (in increasing order) as $\omega_{j}^{k}(T)$ with $j=1,2, \ldots$, we have

$$
\frac{\pi}{2 T}<\omega_{1}^{k}(T)<\frac{3 \pi}{2 T}<\omega_{2}^{k}(T)<\frac{5 \pi}{2 T}<\omega_{3}^{k}(T)<\frac{7 \pi}{2 T}<\omega_{4}^{k}(T)<\ldots
$$

Hence, the spectrum of $K_{\lambda, T}^{(k)}$ is given as

$$
\gamma_{1}^{(k)} \geq \gamma_{2}^{(k)} \geq \gamma_{3}^{(k)} \geq \ldots \geq 0 \text { with } \gamma_{j}^{(k)}=\frac{8 \beta_{k}^{2}}{\alpha_{k}^{2}+\left(\omega_{j}^{k}(T)\right)^{2}}
$$

From (4.10), we now see that the largest eigenvalue $\gamma_{1}$ of $K_{\lambda, T}$ is given as

$$
\gamma_{1}=\max _{k=1, \ldots, d}\left\{\frac{8 \beta_{k}^{2}}{\alpha_{k}^{2}+\left(\omega_{1}^{k}(T)\right)^{2}}\right\}
$$

and that

$$
\sigma\left(K_{\lambda, T}\right)=\left\{\frac{8 \beta_{k}^{2}}{\alpha_{k}^{2}+\left(\omega_{j}^{k}(T)\right)^{2}}: k=1, \ldots, d ; j=1,2, \ldots\right\}
$$


For given $k$ and $j$, the solution of (4.18) associated with $\omega=\omega_{j}^{k}(T)$ is given by $c_{1}=\frac{e^{\mathrm{i} \theta_{j}^{k}}}{2 \mathrm{i}}, c_{2}=$ $\bar{c}_{1}$ with

$$
e^{\mathrm{i} \theta_{j}^{k}}=\frac{-\alpha_{k}+\mathrm{i} \omega_{j}^{k}(T)}{\sqrt{\alpha_{k}^{2}+\left(\omega_{j}^{k}(T)\right)^{2}}} .
$$

Substituting these values in (4.17) we see that the eigenfunction (up to multiplying a constant) associated with the eigenvalue $\gamma_{j}^{(k)}$ is given as

$$
f_{j}^{k}(u)=e^{\mathrm{i}(1+2 \lambda) \beta_{k} u} \sin \left(\omega_{j}^{k}(T) u+\theta_{j}^{k}\right) \cdot U_{k} \in L^{2}\left([0, T] ; \mathbb{C}^{d}\right) .
$$

\section{PROOF OF THE MAIN RESUlt}

5.1. The calculation of $\tilde{\Lambda}$. In this section we characterize the domain of $\tilde{\Lambda}$ and give a formula for $\tilde{\Lambda}(\lambda)$ for $\lambda$ in the domain.

Lemma 5.1. Let $T>0, j \in \mathbb{N}, \alpha<0$. Assume that $\frac{(2 j-1) \pi}{2 T}<\omega<\frac{(2 j+1) \pi}{2 T}$ and that $\theta \in\left[0, \frac{\pi}{2}\right)$ satisfies $e^{\mathrm{i} \theta}=\frac{-\alpha+\mathrm{i} \omega}{\sqrt{\alpha^{2}+\omega^{2}}}$. Then

$$
\frac{1}{2}\left(1-\frac{1}{\pi}\right) T \leq \int_{0}^{T} \sin ^{2}(\omega u+\theta) \mathrm{d} u \leq \frac{1}{2}\left[\left(1+\frac{1}{\pi}\right) T-\frac{1}{\alpha}\right] .
$$

Proof. Since $e^{\mathrm{i} \theta}=\frac{-\alpha+\mathrm{i} \omega}{\sqrt{\alpha^{2}+\omega^{2}}}$ and $\frac{(2 j-1) \pi}{2 T}<\omega<\frac{(2 j+1) \pi}{2 T}$, we have

$$
\frac{\sin 2 \theta}{2 \omega}=\frac{\cos \theta \sin \theta}{\omega}=\frac{-\alpha}{\alpha^{2}+\omega^{2}} \in\left(0,-\frac{1}{\alpha}\right]
$$

and

$$
\left|\frac{\sin 2(\omega T+\theta)}{2 \omega}\right| \leq \frac{1}{2 \omega} \leq \frac{T}{(2 j-1) \pi} \leq \frac{T}{\pi} .
$$

A straightforward calculation gives

$$
\int_{0}^{T} \sin ^{2}(\omega u+\theta) \mathrm{d} u=\frac{1}{2}\left(T-\frac{\sin 2(\omega T+\theta)-\sin 2 \theta}{2 \omega}\right),
$$

which, together with the previous two relations, immediately yields the inequality in the lemma.

Lemma 5.2. Assume the same conditions as in Lemma 5.1. Then

$$
\left|\int_{0}^{T} e^{-\alpha u}\left(e^{2 \alpha u}-e^{2 \alpha T}\right) \sin (\omega u+\theta) \mathrm{d} u\right| \leq \frac{-2 \alpha e^{\alpha T}}{\alpha^{2}+\omega^{2}}+\frac{-2 \omega \alpha}{\left(\alpha^{2}+\omega^{2}\right)^{\frac{3}{2}}} .
$$

Proof. By the following equalities

$\int_{0}^{T} e^{-\alpha u} \sin (\omega u+\theta) \mathrm{d} u=\frac{e^{-\alpha T} \sin \omega T}{\sqrt{\alpha^{2}+\omega^{2}}}, \quad \int_{0}^{T} e^{\alpha u} \sin (\omega u+\theta) \mathrm{d} u=\frac{-e^{\alpha T} \sin (\omega T+2 \theta)+\sin 2 \theta}{\sqrt{\alpha^{2}+\omega^{2}}}$,

we have

$$
\int_{0}^{T} e^{-\alpha u}\left(e^{2 \alpha u}-e^{2 \alpha T}\right) \sin (\omega u+\theta) \mathrm{d} u=\frac{-2 \cos \theta \sin (\omega T+\theta) e^{\alpha T}+\sin 2 \theta}{\sqrt{\alpha^{2}+\omega^{2}}} .
$$

On the other hand, the assumption $e^{\mathrm{i} \theta}=\frac{-\alpha+\mathrm{i} \omega}{\sqrt{\alpha^{2}+\omega^{2}}}$ implies $\cos \theta=-\frac{\alpha}{\sqrt{\alpha^{2}+\omega^{2}}}$ and $\sin \theta=\frac{\omega}{\sqrt{\alpha^{2}+\omega^{2}}}$. This and the previous relation immediately yield the inequality in the lemma. 
Lemma 5.3. Let $\theta \leq \min _{k=1, \ldots, d}\left\{\frac{\alpha_{k}^{2}}{8 \beta_{k}^{2}}\right\}$. For sufficiently large $T$ we have

$$
\left\langle G_{\lambda, T}^{x},\left(\mathrm{Id}-\theta K_{\lambda, T}\right)^{-1} G_{\lambda, T}^{x}\right\rangle_{L^{2}\left([0, T] ; \mathbb{R}^{d}\right)} \leq \frac{1}{\theta^{2}\left(1-\frac{1}{\pi}\right)} \sum_{k=1}^{d}\left|\alpha_{k}\right|\left|\left\langle x, U_{k}\right\rangle_{\mathbb{C}^{d}}\right|^{2} .
$$

Proof. Вy (3.16),

$$
\left\langle G_{\lambda, T}^{x},\left(\mathrm{Id}-\theta K_{\lambda, T}\right)^{-1} G_{\lambda, T}^{x}\right\rangle_{L^{2}\left([0, T] ; \mathbb{R}^{d}\right)}=\sum_{k=1}^{d} \sum_{j=1}^{\infty} \frac{\left|\left\langle G_{\lambda, T}^{x}, f_{j}^{k}\right\rangle_{L^{2}\left([0, T] ; \mathbb{C}^{d}\right)}\right|^{2}}{\left(1-\theta \gamma_{j}^{k}\right)\left\|f_{j}^{k}\right\|_{L^{2}\left([0, T] ; \mathbb{C}^{d}\right)}^{2}},
$$

where $\left\{f_{j}^{k}, j \in \mathbb{N}, k=1, \ldots d\right\}$ is a complete orthonormal system of eigenvectors associated with eigenvalues $\left\{\gamma_{j}^{(k)}\right\}$. Note that when $\beta_{k} \neq 0, f_{j}^{k}$ is given by (4.24). It follows from (4.4) that, when $\beta_{k} \neq 0$,

$$
\begin{aligned}
& \left|\left\langle G_{\lambda, T}^{x}, f_{j}^{k}\right\rangle_{L^{2}\left([0, T] ; \mathbb{C}^{d}\right.}\right| \\
& =\left|\frac{4 \beta_{k}^{2}}{\alpha_{k}}\left\langle x, U_{k}\right\rangle_{\mathbb{C}^{d}} \int_{0}^{T} e^{-\alpha_{k} u}\left(e^{2 \alpha_{k} T}-e^{2 \alpha_{k} u}\right) \sin \left(\omega_{j}^{k}(T) u+\theta_{j}^{k}\right) \mathrm{d} u\right| \\
& \leq \frac{1}{2 \theta}\left|\alpha_{k}\left\langle x, U_{k}\right\rangle_{\mathbb{C}^{d}} \cdot\left[\frac{-2 \alpha_{k} e^{\alpha_{k} T}}{\alpha_{k}^{2}+\left(\omega_{j}^{k}(T)\right)^{2}}+\frac{-2 \omega_{j}^{k}(T) \alpha_{k}}{\left(\alpha_{k}^{2}+\left(\omega_{j}^{k}(T)\right)^{2}\right)^{\frac{3}{2}}}\right]\right|,
\end{aligned}
$$

where the last inequality is by Lemma 5.2. Note that the above inequality is trivially true when $\beta_{k}=0$. By (4.21),

$$
\frac{1}{1-\theta \gamma_{j}^{k}} \leq \frac{1}{1-\frac{\alpha_{k}^{2}}{8 \beta_{k}^{2}} \gamma_{j}^{k}}=\frac{\alpha_{k}^{2}+\left(\omega_{j}^{k}(T)\right)^{2}}{\left(\omega_{j}^{k}(T)\right)^{2}}
$$

The above two bounds and Lemma 5.1 imply that

$$
\begin{aligned}
& \left\langle G_{\lambda, T}^{x},\left(\mathrm{Id}-\theta K_{\lambda, T}\right)^{-1} G_{\lambda, T}^{x}\right\rangle_{L^{2}\left([0, T] ; \mathbb{R}^{d}\right)} \\
& \leq \frac{2}{\theta^{2}\left(1-\frac{1}{\pi}\right)} \sum_{k=1}^{d}\left|\alpha_{k}\left\langle x, U_{k}\right\rangle_{\mathbb{C}^{d}}\right|^{2} \sum_{j=1}^{\infty} \frac{1}{T}\left[\frac{-\alpha_{k} e^{\alpha_{k} T}}{\omega_{j}^{k}(T) \sqrt{\alpha_{k}^{2}+\left(\omega_{j}^{k}(T)\right)^{2}}}+\frac{-\alpha_{k}}{\left(\alpha_{k}^{2}+\left(\omega_{j}^{k}(T)\right)^{2}\right)}\right]^{2} \\
& \leq \frac{2}{\theta^{2}\left(1-\frac{1}{\pi}\right)} \sum_{k=1}^{d}\left|\alpha_{k}\left\langle x, U_{k}\right\rangle_{\mathbb{C}^{d}}\right|^{2} \sum_{j=1}^{\infty} \frac{1}{T}\left[\frac{-2 T \alpha_{k} e^{\alpha_{k} T}}{\pi \sqrt{\alpha_{k}^{2}+\left(\omega_{j}^{k}(T)\right)^{2}}}+\frac{-\alpha_{k}}{\left(\alpha_{k}^{2}+\left(\omega_{j}^{k}(T)\right)^{2}\right)}\right]^{2}
\end{aligned}
$$

where the last inequality is from (4.20). As $T \rightarrow \infty$, the inner sum in the last line has the following limit:

$$
\frac{1}{\pi} \int_{0}^{\infty} \frac{\alpha_{k}^{2}}{\left(\alpha_{k}^{2}+t^{2}\right)^{2}} \mathrm{~d} t=\frac{1}{4\left|\alpha_{k}\right|} .
$$

Indeed, thanks to (4.20) and recalling $\alpha_{k}<0$, we have

$$
\begin{aligned}
& \lim _{T \rightarrow \infty} 2 T e^{\alpha_{k} T}=0, \lim _{T \rightarrow \infty} \sum_{j=1}^{\infty} \frac{1}{T} \frac{\alpha_{k}}{\alpha_{k}^{2}+\left(\omega_{j}^{k}(T)\right)^{2}}=\frac{\pi}{2}, \\
& \lim _{T \rightarrow \infty} \sum_{j=1}^{\infty} \frac{1}{T} \frac{\left(\alpha_{k}\right)^{2}}{\sqrt{\left(\alpha_{k}^{2}+\left(\omega_{j}^{k}(T)\right)^{2}\right)^{3}}}=1, \lim _{T \rightarrow \infty} \sum_{j=1}^{\infty} \frac{1}{T} \frac{\left(\alpha_{k}\right)^{2}}{\left(\alpha_{k}^{2}+\left(\omega_{j}^{k}(T)\right)^{2}\right)^{2}}=\frac{1}{4\left|\alpha_{k}\right|} .
\end{aligned}
$$


Hence, when $T$ is large enough, we have

$$
\sum_{j=1}^{\infty} \frac{1}{T}\left[\frac{-2 T \alpha_{k} e^{\alpha_{k} T}}{\pi \sqrt{\alpha_{k}^{2}+\left(\omega_{j}^{k}(T)\right)^{2}}}+\frac{-\alpha_{k}}{\left(\alpha_{k}^{2}+\left(\omega_{j}^{k}(T)\right)^{2}\right)}\right]^{2} \leq \frac{1}{2\left|\alpha_{k}\right|},
$$

which yields the desired upper bound.

Recall the interval $\mathcal{D}$ introduced in Theorem 2.3.

Lemma 5.4. For $\lambda \in \mathcal{D}$

$$
\tilde{\Lambda}(\lambda)=\lim _{t \rightarrow \infty} \tilde{\Lambda}_{t}(\lambda)=-\frac{1}{2} \sum_{k=1}^{d}\left(\sqrt{\alpha_{k}^{2}-4 \lambda(1+\lambda) \beta_{k}^{2}}+\alpha_{k}\right) .
$$

Moreover, when $\lambda \in \mathcal{D}^{c}$, we have

$$
\lim _{T \rightarrow \infty} \tilde{\Lambda}_{T}(\lambda)=\infty
$$

Proof. For $\lambda \in \mathbb{R}$, denote $\theta=\frac{1}{2} \lambda(1+\lambda)$. Note that $\lambda \in \mathcal{D}$ if and only if $\theta \leq \min _{k=1, \ldots, d}\left\{\frac{\alpha_{k}^{2}}{8 \beta_{k}^{2}}\right\}$. It follows from Theorem 3.3 that when $\theta=\frac{1}{\gamma_{1}} \geq \min _{k=1, \ldots, d}\left\{\frac{\alpha_{k}^{2}+\left(\omega_{1}^{k}(T)\right)^{2}}{8 \beta_{k}^{2}}\right\}$,

$$
\frac{1}{T} \log \mathbb{E}^{x} \exp \left(\theta \int_{0}^{T}\left|Z_{\lambda, t}\right|^{2} \mathrm{~d} t\right)=\infty, \quad \text { for all } x \in \mathbb{R}^{d}, \text { and } \quad T>0,
$$

and thus (5.3) also holds true with $\mathbb{E}^{x}$ replaced by $\mathbb{E}^{\mu}$. This shows that

$$
\tilde{\Lambda}_{T}(\lambda)=\infty \quad \text { whenever } \frac{1}{2} \lambda(1+\lambda) \geq \min _{k=1, \ldots, d}\left\{\frac{\alpha_{k}^{2}+\left(\omega_{1}^{k}(T)\right)^{2}}{8 \beta_{k}^{2}}\right\} .
$$

The second relation in the lemma is now immediate.

We now prove the first statement in the lemma. We need to show that when $\theta \leq \min _{k=1, \ldots, d}\left\{\frac{\alpha_{k}^{2}}{8 \beta_{k}^{2}}\right\}$

$$
\frac{1}{T} \log \mathbb{E}^{\mu} \exp \left(\theta \int_{0}^{T}\left|Z_{\lambda, t}\right|^{2} \mathrm{~d} t\right)
$$

converges to the right side of (5.2). By Theorem 3.3

$$
\begin{aligned}
\mathbb{E}^{\mu} & \exp \left(\theta \int_{0}^{T}\left|Z_{\lambda, t}\right|^{2} \mathrm{~d} t\right) \\
= & \frac{1}{\sqrt{\operatorname{det}\left(\operatorname{Id}-\theta K_{\lambda, T}\right)}} \\
& \times \int_{\mathbb{R}^{d}} \exp \left[\theta S_{0}^{x}(T)-\frac{\theta}{2} \operatorname{tr}\left(K_{\lambda, T}\right)+\frac{\theta^{2}}{2}\left\langle G_{\lambda, T}^{x},\left(\operatorname{Id}-\theta K_{\lambda, T}\right)^{-1} G_{\lambda, T}^{x}\right\rangle_{L^{2}\left([0, T] ; \mathbb{R}^{d}\right)}\right] \mu(d x),
\end{aligned}
$$

Thus, using (3.2),

$$
\frac{1}{T} \log \mathbb{E}^{\mu} \exp \left(\theta \int_{0}^{T}\left|Z_{\lambda, t}\right|^{2} \mathrm{~d} t\right)=I_{1}+I_{2}+I_{3}
$$

where $I_{3}=-\frac{1}{2 T} \log \left[\operatorname{det}\left(\operatorname{Id}-\theta K_{\lambda, T}\right)\right]$,

$$
I_{1}=-\frac{\theta}{2 T} \operatorname{tr}\left(K_{\lambda, T}\right)+\frac{\theta}{T} \int_{0}^{T} \operatorname{tr}\left[N^{\prime} e^{u M} N\right](T-u) \mathrm{d} u,
$$


and

$I_{2}=\frac{1}{T} \log \int_{\mathbb{R}^{d}} \exp \left[\left|\left(\int_{0}^{T} e^{s M} \mathrm{~d} s\right)^{\frac{1}{2}} N x\right|^{2} \theta+\frac{\theta^{2}}{2}\left\langle G_{\lambda, T}^{x},\left(\mathrm{Id}-\theta K_{\lambda, T}\right)^{-1} G_{\lambda, T}^{x}\right\rangle_{L^{2}\left([0, T] ; \mathbb{R}^{d}\right)}\right] \mu(d x)$

Observe that the following relations hold:

$$
\int_{0}^{T} e^{u M} \mathrm{~d} u=M^{-1}\left(e^{M T}-\mathrm{Id}\right), \quad \int_{0}^{T} e^{u M}(T-u) \mathrm{d} u=M^{-2}\left(e^{M T}-\mathrm{Id}\right)-T M^{-1} .
$$

Since $M$ is negative definite, $\left\|e^{M t}\right\| \leq 1$ for all $t \geq 0$, and thus

$$
\lim _{T \rightarrow \infty} \frac{1}{T} \int_{0}^{T} \operatorname{tr}\left[N^{\prime} e^{u M} N\right](T-u) \mathrm{d} u=-\operatorname{tr}\left(N^{\prime} M^{-1} N\right) .
$$

On the other hand, (3.7) implies $\lim _{T \rightarrow \infty} \frac{1}{T} \operatorname{tr}\left(K_{\lambda, T}\right)=-2 \operatorname{tr}\left(N^{\prime} M^{-1} N\right)$, which, together with the previous limit, yields

$$
\lim _{T \rightarrow \infty} I_{1}=0
$$

Recalling that the density of $\mu$ is given by (2.11), the relation (4.3) and Lemma 5.3 imply that

$$
\begin{aligned}
I_{2} & \leq \frac{1}{T} \log \int_{\mathbb{R}^{d}}(2 \pi)^{-\frac{d}{2}}|\operatorname{det}(M)|^{\frac{1}{2}} \exp \left[\sum_{k=1}^{d}\left(\frac{\left|\alpha_{k}\right|}{4}+\frac{\left|\alpha_{k}\right|}{2\left(1-\frac{1}{\pi}\right)}\right)\left|\left\langle x, U_{k}\right\rangle_{\mathbb{C}^{d}}\right|^{2}\right] \exp \left(\frac{x^{\prime} M x}{2}\right) \mathrm{d} x \\
& =\frac{1}{T} \log \int_{\mathbb{R}^{d}}(2 \pi)^{-\frac{d}{2}}|\operatorname{det}(M)|^{\frac{1}{2}} \exp \left[\left(\frac{3}{4}-\frac{1}{2\left(1-\frac{1}{\pi}\right)}\right) \sum_{k=1}^{d} \alpha_{k}\left|y_{k}\right|^{2}\right] \mathrm{d} x,
\end{aligned}
$$

where the last inequality uses the fact that $U^{*} M U=\operatorname{diag}\left\{2 \alpha_{1}, \ldots, 2 \alpha_{d}\right\}$ by (4.2), and $\exp \left(\frac{x^{\prime} M x}{2}\right)=$ $\exp \left(\sum_{k=1}^{d} \alpha_{k}\left|y_{k}\right|^{2}\right)$ with $y_{k}=\left\langle x, U_{k}\right\rangle_{\mathbb{C}^{d}}$. Since $\frac{3}{4}-\frac{1}{2\left(1-\frac{1}{\pi}\right)}>0$ and $\alpha_{k}<0$, we have that, $I_{2}<\frac{c}{T}$ with a constant $c>0$ depending only on $M$. Thus $\lim _{T \rightarrow \infty} I_{2}=0$.

For $I_{3}$, by Theorem 3.3 and (4.23) we have

$$
\begin{aligned}
I_{3} & =-\frac{1}{2 T} \sum_{j=1}^{\infty} \sum_{k=1}^{d} \log \left(1-\theta \gamma_{j}^{(k)}\right) \\
& =-\frac{1}{2 T} \sum_{j=1}^{\infty} \sum_{k=1}^{d} \log \left(1-\frac{8 \theta \beta_{k}^{2}}{\alpha_{k}^{2}+\left(\omega_{j}^{k}(T)\right)^{2}}\right) \\
& \rightarrow-\frac{1}{2} \int_{0}^{\infty} \sum_{k=1}^{d} \log \left(1-\frac{8 \theta \beta_{k}^{2}}{\alpha_{k}^{2}+\pi^{2} x^{2}}\right) \mathrm{d} x
\end{aligned}
$$

as $T \rightarrow \infty$, where the convergence on the last line follows from (4.20).

The result now follows on observing that for $b>0$ and $b \geq a$,

$$
\int_{0}^{\infty} \log \left(1-\frac{a}{b+x^{2}}\right) d x=(\sqrt{b-a}-\sqrt{b}) \pi .
$$


5.2. Proofs of Theorems 2.1 and 2.3. For $n \in \mathbb{N}$, define

$$
\tau_{n}=\inf \left\{t \geq 0:\left|X_{t}\right| \geq n\right\}, \quad X_{t}^{n}=X_{t} 1_{\left\{t \leq \tau_{n}\right\}} .
$$

Clearly, $\lim _{n \rightarrow \infty} \tau_{n}=\infty$ a.s. For $\lambda \in \mathbb{R}$, let

$$
\mathrm{d} W_{t}=\mathrm{d} B_{t}-\lambda N X_{t}^{n} \mathrm{~d} t
$$

and

$$
\begin{aligned}
\mathcal{E}_{t}^{n}(\lambda) & =\exp \left\{-\frac{\lambda^{2}}{2} \int_{0}^{t}\left|N X_{s}^{n}\right|^{2} \mathrm{~d} s+\lambda \int_{0}^{t}\left(N X_{s}^{n}\right)^{\prime} \mathrm{d} B_{s}\right\} \\
& =\exp \left\{-\frac{\lambda^{2}}{2} \int_{0}^{t \wedge \tau_{n}}\left|N X_{s}\right|^{2} \mathrm{~d} s+\lambda \int_{0}^{t \wedge \tau_{n}}\left(N X_{s}\right)^{\prime} \mathrm{d} B_{s}\right\} .
\end{aligned}
$$

Recall $\tilde{\Lambda}$ and $\Lambda$ in Section 2 . Denote the probability space on which the stationary process $X_{t}$ and the Brownian motion $B_{t}$ are defined as $(\Omega, \mathcal{F}, \mathbb{P})$. Let $\mathcal{F}_{t}$ be the filtration generated by $X_{0}$ and $\left\{B_{t}\right\}$, namely $\mathcal{F}_{t}=\sigma\left\{X_{0}, B_{s}, 0 \leq s \leq t\right\}$. Let

$$
\Lambda_{t}^{n}(\lambda)=\frac{1}{t} \log \mathbb{E} \exp \left\{\lambda \int_{0}^{t \wedge \tau_{n}}\left(N X_{s}\right)^{\prime} \mathrm{d} B_{s}+\frac{\lambda}{2} \int_{0}^{t \wedge \tau_{n}}\left|N X_{s}\right|^{2} \mathrm{~d} s\right\} .
$$

We now prove (2.18).

Proposition 5.5. We have $\mathcal{D}_{\tilde{\Lambda}}=\mathcal{D}_{\Lambda}$ and $\tilde{\Lambda}=\Lambda$.

Proof. Step 1. The relation between $X_{t}$ and $Y_{\lambda, t}$ : Since $\left|X_{t}^{n}\right| \leq n$ for all $t$, Novikov's condition (cf. [25, 3.5.D]) clearly holds for the exponential supermartingale $\mathcal{E}_{t}^{n}(\lambda)$. Thus Girsanov theorem yields that $\left(W_{t}\right)_{t \geq 0}$ is a standard $d$-dimensional Brownian motion under the measure $\mathbb{P}_{W}^{n}$ which is uniquely determined by

$$
\left.\frac{\mathrm{d} \mathbb{P}_{W}^{n}}{\mathrm{dP}}\right|_{\mathcal{F}_{t}}=\mathcal{E}_{t}^{n}(\lambda), \quad t \geq 0
$$

and equation (2.13) can be rewritten as

$$
\mathrm{d} X_{t}=A X_{t} \mathrm{~d} t+\lambda N X_{t} 1_{\left\{t \leq \tau_{n}\right\}} \mathrm{d} t+\mathrm{d} W_{t} .
$$

If one defines $Y_{\lambda, t}$ on $\left(\Omega, \mathcal{F}, \mathbb{P}_{W}^{n}\right)$ as

$$
\mathrm{d} Y_{\lambda, t}=D_{\lambda} Y_{\lambda, t} \mathrm{~d} t+\mathrm{d} W_{t}, Y_{\lambda, 0}=X_{0},
$$

then $\left\{Y_{\lambda, t}\right\}$ (under $\mathbb{P}_{W}^{n}$ ) has the same law as the process in (2.14) under the stationary measure $\mathbb{P}^{\mu}$ considered there. Also, $Z_{\lambda, s}=N Y_{\lambda, s}=N X_{s}$ for $0 \leq s \leq \tau_{n} \wedge t$. Thus

$$
\begin{aligned}
\Lambda_{t}^{n}(\lambda) & =\frac{1}{t} \log \mathbb{E} \exp \left\{\lambda \int_{0}^{t \wedge \tau_{n}}\left(N X_{s}\right)^{\prime} \mathrm{d} B_{s}+\frac{\lambda}{2} \int_{0}^{t \wedge \tau_{n}}\left|N X_{s}\right|^{2} \mathrm{~d} s\right\} \\
& =\frac{1}{t} \log \mathbb{E}^{\mathbb{P}_{W}^{n}} \exp \left\{\frac{1}{2} \lambda(1+\lambda) \int_{0}^{t \wedge \tau_{n}}\left|N X_{s}\right|^{2} \mathrm{~d} s\right\} \\
& =\frac{1}{t} \log \mathbb{E}^{\mu} \exp \left\{\frac{1}{2} \lambda(1+\lambda) \int_{0}^{t \wedge \tau_{n}}\left|Z_{\lambda, s}\right|^{2} \mathrm{~d} s\right\} .
\end{aligned}
$$

By Fatou's lemma and monotone convergence theorem, we have

$$
\Lambda_{t}(\lambda) \leq \lim _{n \rightarrow \infty} \Lambda_{t}^{n}(\lambda)=\tilde{\Lambda}_{t}(\lambda) .
$$

Step 2. $\mathcal{D}_{\tilde{\Lambda}}^{\circ} \subset \mathcal{D}_{\Lambda}^{\circ}$ : Recall from Lemma 5.4 the domain of $\tilde{\Lambda}$ is a finite closed interval, we denote this interval by $[a, b]$ and see that $-\infty<a<0<b<\infty$. Clearly $0 \in \mathcal{D}_{\tilde{\Lambda}} \cap \mathcal{D}_{\Lambda}$. 
Suppose now that $\lambda \in(0, b)$. Fix a $\bar{\lambda} \in(\lambda, b)$. We have, for $t>0$,

$$
\Lambda_{t}^{n}(\bar{\lambda})=\frac{1}{t} \log \mathbb{E}^{\mu} \exp \left\{\frac{1}{2} \bar{\lambda}(1+\bar{\lambda}) \int_{0}^{t \wedge \tau_{n}}\left|Z_{\lambda, s}\right|^{2} \mathrm{~d} s\right\} \leq \tilde{\Lambda}_{t}(\bar{\lambda})<\infty .
$$

Because $0<\lambda<\bar{\lambda}$, the above inequality implies a uniform integrability (with respect to $n$ ) for $\exp \left\{\lambda \int_{0}^{t \wedge \tau_{n}}\left(N X_{s}\right)^{\prime} \mathrm{d} B_{s}+\frac{\lambda}{2} \int_{0}^{t \wedge \tau_{n}}\left|N X_{s}\right|^{2} \mathrm{~d} s\right\}$, and thus

$$
\begin{aligned}
& \mathbb{E}^{\mu} \exp \left\{\lambda \int_{0}^{t}\left(N X_{s}\right)^{\prime} \mathrm{d} B_{s}+\frac{\lambda}{2} \int_{0}^{t}\left|N X_{s}\right|^{2} \mathrm{~d} s\right\} \\
= & \lim _{n \rightarrow \infty} \mathbb{E}^{\mu} \exp \left\{\lambda \int_{0}^{t \wedge \tau_{n}}\left(N X_{s}\right)^{\prime} \mathrm{d} B_{s}+\frac{\lambda}{2} \int_{0}^{t \wedge \tau_{n}}\left|N X_{s}\right|^{2} \mathrm{~d} s\right\} .
\end{aligned}
$$

Hence,

$$
\Lambda_{t}(\lambda)=\lim _{n \rightarrow \infty} \Lambda_{t}^{n}(\lambda)=\tilde{\Lambda}_{t}(\lambda) \text { for } \lambda \in(0, b)
$$

Similarly, we have

$$
\Lambda_{t}(\lambda)=\lim _{n \rightarrow \infty} \Lambda_{t}^{n}(\lambda)=\tilde{\Lambda}_{t}(\lambda) \text { for } \lambda \in(a, 0) .
$$

Hence, $\mathcal{D}_{\tilde{\Lambda}}^{\circ} \subset \mathcal{D}_{\Lambda}^{\circ}$ and

$$
\tilde{\Lambda}(\lambda)=\Lambda(\lambda) \text { for } \lambda \in \mathcal{D}_{\tilde{\Lambda}}^{\circ} \text {. }
$$

Step 3. $\mathcal{D}_{\Lambda} \subset \mathcal{D}_{\tilde{\Lambda}}$ : We argue via contradiction. Suppose there exists a $\lambda^{*} \in \mathcal{D}_{\Lambda}$ such that $\lambda^{*} \notin \mathcal{D}_{\tilde{\Lambda}}$, i.e. $\lambda^{*} \notin[a, b]$. Assume that $\lambda^{*}>b$; the case $\lambda^{*}<a$ can be handled similarly. Recalling the definition of $[a, b]$ (see Lemma 5.4), we see that $\theta^{*}=\frac{1}{2} \lambda^{*}\left(1+\lambda^{*}\right)>\min _{k=1, \ldots, d}\left\{\frac{\alpha_{k}^{2}}{8 \beta_{k}^{2}}\right\}$. Since $\lim _{T \rightarrow \infty} \omega_{1}^{k}(T)=0$ for each $k=1, \ldots, d$, we can choose $T$ sufficiently large so that $\theta^{*}>\hat{\theta}$ with $\hat{\theta}=\min _{k=1, \ldots, d}\left\{\frac{\alpha_{k}^{2}+\left(\omega_{1}^{k}(T)\right)^{2}}{8 \beta_{k}^{2}}\right\}$. Also, since $\Lambda\left(\lambda^{*}\right)<\infty$, by choosing $T$ larger if needed, we can assume that $\Lambda_{T}\left(\lambda^{*}\right)<\infty$. Choose an increasing sequence $\left\{\lambda_{n}\right\}_{n \geq 1}$ such that $\lambda_{n}>b$ and $\theta_{n} \uparrow \theta^{*}$ with $\theta_{n}=\frac{1}{2} \lambda_{n}\left(1+\lambda_{n}\right)$. Clearly, $\lambda_{n}<\lambda^{*}$, and since $\lim \theta_{n}>\hat{\theta}$, by (5.4) we know

$$
\lim _{n \rightarrow \infty} \tilde{\Lambda}_{T}\left(\lambda_{n}\right)=\infty .
$$

On the other hand, since $\lambda_{n}<\lambda^{*}$, by the same argument as in the proof of (5.10), we get $\tilde{\Lambda}_{T}\left(\lambda_{n}\right)=\Lambda_{T}\left(\lambda_{n}\right)$ and thus

$$
\lim _{n \rightarrow \infty} \Lambda_{T}\left(\lambda_{n}\right)=\infty \text {. }
$$

By Hölder inequality we get $\Lambda_{T}\left(\lambda^{*}\right) \geq \frac{\lambda^{*}}{\lambda_{n}} \Lambda_{T}\left(\lambda_{n}\right)$ for all $\lambda_{n}$, and hence $\Lambda_{T}\left(\lambda^{*}\right)=\infty$. But this is a contradiction since $T$ is chosen such that $\Lambda_{T}\left(\lambda^{*}\right)<\infty$. Thus we have that $\mathcal{D}_{\Lambda} \subset \mathcal{D}_{\tilde{\Lambda}}$.

Step 4. $\mathcal{D}_{\Lambda}=\mathcal{D}_{\tilde{\Lambda}}$ and $\tilde{\Lambda}=\Lambda$ : From the previous two steps, we clearly see that $(a, b) \subset$ $\mathcal{D}_{\Lambda} \subset[a, b]$ and $\tilde{\Lambda}(\lambda)=\Lambda(\lambda)$ for $\lambda \in(a, b)$. To conclude the proof, we only need to prove $\Lambda(a)=\tilde{\Lambda}(a)$ and $\Lambda(b)=\tilde{\Lambda}(b)$. For a small $\varepsilon>0$, by (5.7), (5.10) and an application of Hölder inequality to $\Lambda_{t}(b-\varepsilon)$, we have

which leads to

$$
\tilde{\Lambda}_{t}(b-\varepsilon)=\Lambda_{t}(b-\varepsilon) \leq \frac{b-\varepsilon}{b} \Lambda_{t}(b) \leq \frac{b-\varepsilon}{b} \tilde{\Lambda}_{t}(b), \quad t>0,
$$

$$
\tilde{\Lambda}(b-\varepsilon) \leq \frac{b-\varepsilon}{b} \liminf _{t \rightarrow \infty} \Lambda_{t}(b) \leq \frac{b-\varepsilon}{b} \limsup _{t \rightarrow \infty} \Lambda_{t}(b) \leq \frac{b-\varepsilon}{b} \tilde{\Lambda}(b) .
$$


Since $\lim _{\varepsilon \rightarrow 0+} \tilde{\Lambda}(b-\varepsilon)=\tilde{\Lambda}(b)$ by Lemma 5.4, we have $\Lambda(b)=\tilde{\Lambda}(b)$. Similarly we get $\Lambda(a)=$ $\tilde{\Lambda}(a)$.

In order to verify conditions of Gärtner-Ellis theorem, define

$$
F(\ell)=\frac{1}{2} \sum_{k=1}^{d}\left(\sqrt{\alpha_{k}^{2}-\ell \beta_{k}^{2}}+\alpha_{k}\right), \quad \ell \in\left(-\infty, \min _{k=1, \ldots, d}\left\{\frac{\alpha_{k}^{2}}{\beta_{k}^{2}}\right\}\right] .
$$

It is easy to check that

$$
F^{\prime}(\ell)=-\frac{1}{4} \sum_{k=1}^{d} \frac{\beta_{k}^{2}}{\sqrt{\alpha_{k}^{2}-\ell \beta_{k}^{2}}}, \quad \ell \in\left(-\infty, \min _{k=1, \ldots, d}\left\{\frac{\alpha_{k}^{2}}{\beta_{k}^{2}}\right\}\right) .
$$

Note that $\left|F^{\prime}(\ell)\right| \rightarrow \infty$ as $\ell \rightarrow \min _{k=1, \ldots, d}\left\{\frac{\alpha_{k}^{2}}{\beta_{k}^{2}}\right\}-$.

We can now complete the proofs of our main results.

Proofs of Theorems 2.1 and 2.3. The first relation in Theorem 2.3 follows immediately from Proposition 5.5 and Lemma 5.4. Now we prove Theorem 2.1 and the Cohen-Gallavotti symmetry properties. From the previous proposition, Assumption 2.3.2 of [5] is satisfied. Let

$$
\ell(\lambda)=4 \lambda(1+\lambda) .
$$

Then by Proposition 5.5 and Lemma 5.4, we have that for all $\lambda \in \mathcal{D}_{\Lambda}$,

$$
\begin{aligned}
\Lambda(\lambda) & =-F(\ell(\lambda)), \\
\Lambda^{\prime}(\lambda) & =(1+2 \lambda) \sum_{k=1}^{d} \frac{\beta_{k}^{2}}{\sqrt{\alpha_{k}^{2}-\ell(\lambda) \beta_{k}^{2}}} .
\end{aligned}
$$

Hence, $\Lambda^{\prime}(\lambda)$ exists for all $\lambda \in \mathcal{D}_{\Lambda}^{\circ}$ and

$$
\lim _{\lambda \rightarrow \partial \mathcal{D}_{\Lambda}}\left|\Lambda^{\prime}(\lambda)\right|=\infty
$$

From this it follows that $\Lambda$ is a lower semicontinuous function on $\mathbb{R}$ which is essentially smooth in the sense of [5, Definition 2.3.5]. Thus by Gärtner-Ellis Theorem [5, Section 2.3], we immediate obtain that EPR $e_{p}(t)$ satisfies an LDP with rate function $I$ (in particular, $I$ has compact level sets) given as

$$
\begin{aligned}
I(x) & =\sup \left\{x \lambda+F(\ell(\lambda)): \lambda \in \mathcal{D}_{\Lambda}\right\} \\
& =\sup \left\{x \lambda(\ell)+F(\ell):-1 \leq \ell \leq \min _{k=1, \ldots, d}\left\{\frac{\alpha_{k}^{2}}{\beta_{k}^{2}}\right\}\right\},
\end{aligned}
$$

where $F(\ell)$ is given as (5.13) and $\lambda(\ell)$ is an inverse function of $\ell=4 \lambda(1+\lambda)$ defined as $\lambda(\ell)=\frac{\sqrt{\ell+1}-1}{2}$ for $x \geq 0$ and $\lambda(\ell)=\frac{-\sqrt{\ell+1}-1}{2}$ for $x<0$.

When $x=0$, it is clear that $I(0)=F(-1)$ since $F(\ell)$ is strictly decreasing on $\left[-1, \min _{k=1, \ldots, d}\left\{\frac{\alpha_{k}^{2}}{\beta_{k}^{2}}\right\}\right]$.

When $x \neq 0$, by differentiating the function $s(\ell):=x \lambda(\ell)+F(\ell)$, we have that the unique zero point $\ell_{0}(x)$ of $s^{\prime}(\ell)$ is given as the solution of the following equation:

$$
|x|=\sqrt{1+\ell} \sum_{k=1}^{d} \frac{\beta_{k}^{2}}{\sqrt{\alpha_{k}^{2}-\ell \beta_{k}^{2}}}, \quad-1 \leq \ell<\min _{k=1, \ldots, d}\left\{\frac{\alpha_{k}^{2}}{\beta_{k}^{2}}\right\} .
$$

Substituting it into $s(\ell)$, we obtain (2.6).

Finally, the Cohen-Gallavotti symmetry properties in (2.10) are immediate from the explicit expressions for $\Lambda$ and $I$ in (2.9) and (2.6), respectively. 


\section{APPENDIX}

Proof of the claim in (1.5) Since $A \in \mathbb{R}^{d \times d}$ is normal, there is a real orthogonal matrix $P$ (cf. [18, Theorem 2.5.8]) such that

$$
P^{\prime} A P=\operatorname{diag}\left\{d_{1}, \ldots, d_{m}, E_{1}, E_{2}, \cdots, E_{k}\right\},
$$

where for $1 \leq i \leq m, d_{i}$ is a real number and for $1 \leq j \leq k, E_{j}$ is a real $2 \times 2$ matrix of the form,

$$
E_{j}=\left[\begin{array}{cc}
e_{j} & \tilde{e}_{j} \\
-\tilde{e}_{j} & e_{j}
\end{array}\right]
$$

and $m+2 k=d$, where $e_{j} \in \mathbb{R}, \tilde{e}_{j} \in \mathbb{R}$ with $\tilde{e}_{j} \neq 0$. From (6.1) and assumption (A), we see that

(6.2)

$$
M:=A+A^{\prime} \text { has eigenvalues }\left\{2 d_{i}, 2 e_{j}, 1 \leq i \leq m, 1 \leq j \leq k\right\} \text { and is negative definite. }
$$

Define

$$
\hat{A}=\operatorname{diag}\left\{d_{1}, \ldots, d_{m}, e_{1}+\mathrm{i} \tilde{e}_{1}, e_{1}-\mathrm{i} \tilde{e}_{1}, \ldots, e_{k}+\mathrm{i} \tilde{e}_{k}, e_{k}-\mathrm{i} \tilde{e}_{k}\right\}
$$

Then there is a complex unitary matrix $U$ (cf. [18, Theorems 2.5.3 and 2.5.8]), such that

$$
U^{*} A U=\operatorname{diag}\{\hat{A}\}, .
$$

By our assumption in (1.2) and [18, Theorems 2.5.5 and 2.5.6] it follows that the above unitary matrix $U$ and the diffusion matrix $Q$ satisfy

$$
U^{*} Q U=\operatorname{diag}\left\{q_{1}, \ldots, q_{d}\right\}
$$

where $q_{k}>0$ are the eigenvalues of $Q$. It is easy to check using (4.1) and (6.4) that

$$
A^{\prime} Q=Q A^{\prime}, \quad Q^{\frac{1}{2}} A=A Q^{\frac{1}{2}}, \quad Q^{\frac{1}{2}} A^{\prime}=A^{\prime} Q^{\frac{1}{2}} .
$$

The statement in (1.5) is immediate from this.

Acknowledgement. Research of AB is supported in part by the National Science Foundation (DMS-1814894 and DMS-1853968). Research of YC is supported in part by NSFC Grants (11871079, 11961033). Research of LX is supported in part by Macao S.A.R grant FDCT 0090/2019/A2 and University of Macau grant MYRG2018-00133-FST.

\section{REFERENCES}

[1] Balescu, R. (1997). Statistical dynamics: Matter out of equilibrium, Imperial College Press, London.

[2] L. Bertini and G. Di Gesù (2015). Small noise asymptotic of the Gallavotti-Cohen functional for diffusion processes, ALEA, Lat. Am. J. Probab. Math. Stat., 12: 743-763.

[3] Chen, X. (2008) Intersection local times: Large deviations and laws of the iterated logarithm. Asymptotic theory in probability and statistics with applications, Adv. Lect. Math. (ALM), 2, Int. Press, Somerville, MA.

[4] Chen, Y., Ge, H., Xiong, J. and Xu, L. (2016). The large deviation principle and steady-state FT for the entropy production rate of a stochastic process in magnetic fields, J. Math. Phys. 57, 073302.

[5] Dembo A., Zeitouni O. (2000). Large Deviations Techniques and Applications, Springer-Verlag, New York.

[6] Donsker, M.D. and Varadhan, S. R. S. (1975, 1975, 1976, 1983). Asymptotic evaluation of certain Markov process expectations for large time, I-IV, Comm. Pure Appl. Math., 28, 1-47,279-301; 29, 389-461; 36,183212.

[7] Endres, R.G. Entropy production selects nonequilibrium states in multistable systems. Sci Rep 7, 14437 (2017).

[8] Evans, D.J., Cohen, E.G.D. and Morriss, G.P. (1993). Probability of second law violation in steady flows. Phys. Rev. Lett. 71, 2401-2404.

[9] Freidlin M.I. and Wentzell, A. D. (1984). Random Perturbations of Dynamical Systems. Springer-Verlag, New York. 
[10] Friz,P.K., Gassiat, P. and Lyons,T. (2015). Physical Brownian motion in a magnetic field as a rough path. Trans. Amer. Math. Soc. 367, 7939-7955.

[11] Gallavotti, G. and Cohen, E.G.D. (1995). Dynamical ensembles in stationary states. J. Statist. Phys. 80, 931-970

[12] Gao, F. and Jiang, H. (2017). Deviation inequalities for quadratic Wiener functionals and moderate deviations for parameter estimators. Sci. China Math. 60, no. 7, 1181-1196.

[13] Garćia-Garćia R. (2012) Nonadiabatic entropy production for non-Markov dynamics. Phys. Rev. E 86 031117.

[14] Garling, D.J.H. (2007). Inequalities:A Journey into Linear Analysis. Cambridge University Press, 2007.

[15] Ge, H., Qian, M. and Qian, H. (2012). Stochastic theory of nonequilibrium steady states (Part II): Applications in chemical biophysics. Phys. Rep. 510, 87-118

[16] Grasselli, M. R. and Hurd, T. R (2005). Wiener chaos and the Cox-Ingersoll-Ross model. Proc R Soc Lond Ser A Math Phys Eng Sci, 461, 459-479.

[17] Harris, R.J. and Schuetz, G. M. (2007). Fluctuation theorems for stochastic dynamics. J. Stat. Mech.,P07020.

[18] Horn R. A., Johnson C. R. (2013). Matrix Analysis (2nd Edition), Cambridge University Express.

[19] Itô K. (1953). Complex multiple Wiener integral, Japan J.Math. 22, 63-86 Reprinted in: Kiyosi Itô selected papers, Edited by Daniel W. Stroock, S.R.S. Varadhan, Springer-Verlag, 1987.

[20] V. Jakšić, V. Nersesyan, C.-A. Pillet, A. Shirikyan. Large deviations and Gallavotti-Cohen principle for dissipative PDEs with rough noise. Comm. Math. Phys. 336 (2015), no. 1, 131-170.

[21] V. Jakšić, C.-A. Pillet, A. Shirikyan. Entropic fluctuations in Gaussian dynamical systems. Rep. Math. Phys. 77 (2016), no. 3, 335-376.

[22] V. Jakšić, C.-A. Pillet, A. Shirikyan. Entropic fluctuations in thermally driven harmonic networks. J. Stat. Phys. 166 (2017), no. 3-4, 926-1015.

[23] Jiang, D. Q., Qian, M. and Qian, M. P. (2004). Mathematical Theory of Nonequilibrium Steady States - on the Frontier of Probability and Dynamical Systems. (Lect. Notes Math.1833) Berlin: Springer-Verlag

[24] Jiang D.-Q., Qian M., Zhang F.-X. (2003). Entropy production fluctuations of finite Markov chains. Journal of Mathematical Physics, 44(9), 4176.

[25] Karatzas, I., Shreve, S. (1991). Brownian Motion and Stochastic Calculus. Springer-Verlag, New York.

[26] Kifer, Y. (1990). Large deviations in dynamical systems and stochastic processes. Trans. Amer. Math. Soc. 321, no. 2, 505-524.

[27] Kurchan, J. (1998). Fluctuation theorem for stochastic dynamics. J. Phys. A: Math. Gen. 31, 3719-3729

[28] Landi G. T. , Tomé T. , de Oliveira M. J. (2013). Entropy production in linear Langevin systems. J. Phys. A 46, 395001.

[29] Lebowitz, J.L. and Spohn, H. (1999). A Gallavotti-Cohen-type symmetry in the large deviation functional for stochastic dynamics. J. Statist. Phys. 95, 333-365.

[30] Qian, H. (2001). Mathematical formalism for isothermal linear irreversibility. Proc. Roy. Soc. A, 457, 16451655.

[31] Qian, M.P., Qian, M. and Gong, G.L. (1991). The reversibility and the entropy production of Markov processes. Probability theory and its applications in China, 255-261, Contemp. Math., 118, Amer. Math. Soc., Providence, RI.

[32] Rey-Bellet, L. and Thomas, L. E. (2002). Fluctuations of the entropy production in anharmonic chains. Ann. Henri Poicaré 3 (3), 483-502.

[33] Santos, J.P., Céleri, L.C., Landi, G.T. (2019). The role of quantum coherence in non-equilibrium entropy production. npj Quantum Inf 5, 23.

[34] Santos, J. P., Landi, G. T., Paternostro, M. (2017). Wigner entropy production rate. Physical Review Letters, 118, 220601.

[35] Seara, D.S., Yadav, V., Linsmeier, I. (2018). Entropy production rate is maximized in non-contractile actomyosin. Nat Commun 9, 4948.

[36] Tomé T. , de Oliveira M. J. (2012) Entropy Production in Nonequilibrium Systems at Stationary States. Phys. Rev. Lett. 108, 020601.

[37] Wang R., Xu L. 2015, Asymptotics of the entropy production rate for d-dimensional Ornstein-Uhlenbeck processes, J. Stat. Phys., no. 5, 1336-1353

[38] Whitfield J. (2005). Complex systems: Order out of chaos. Nature 436 (7053): 905-907.

[39] $\mathrm{L} . \mathrm{Wu}$, Large and moderate deviations and exponential convergence for stochastic damping Hamiltionian systems, Stochastic Proc. Appl. 91 (2001), 205-238.

[40] Zhang, X. J., Qian, H. and Qian, M. (2012). Stochastic theory of nonequilibrium steady states and its applications (Part I). Phys. Rep. 510, 1-86 


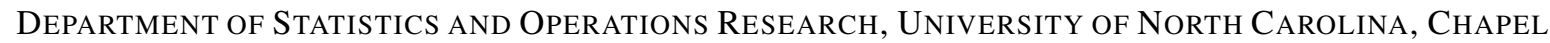
HiLl, NC 27599, UNiTED STATES

E-mail address: budhirajeemail.unc.edu

College of Mathematics and Information Science, JiangXi Normal University, Nanchang, JiANGXI, 330022, P. R. CHINA

E-mail address: zhishi@pku.org.cn

1. Department of Mathematics, Faculty of Science and Technology University of Macau Av. Padre Tomás Pereira, Taipa Macau, China, 2. UMacau Zhuhai Research Institute, China E-mail address: li huxu@um. edu.mo 